\title{
Axisymmetric Schwarzschild models of an isothermal axisymmetric mock dwarf spheroidal galaxy
}

\author{
Jorrit H. J. Hagen, Amina Helmi, and Maarten A. Breddels
}

\begin{abstract}
Kapteyn Astronomical Institute, University of Groningen, PO Box 800, 9700 AV Groningen, The Netherlands e-mail: hagen@astro.rug.nl
\end{abstract}

Received 27 June 2019 / Accepted 7 October 2019

\begin{abstract}
Aims. The goal of this work is to test the ability of Schwarzschild's orbit superposition method to measure the mass content, scale radius, and shape of a flattened dwarf spheroidal galaxy. Until now, most dynamical model efforts have assumed that dwarf spheroidal galaxies and their host halos are spherical.

Methods. We used an Evans model (1993, MNRAS, 260, 191) to construct an isothermal mock galaxy whose properties somewhat resemble those of the Sculptor dwarf spheroidal galaxy. This mock galaxy contains flattened luminous and dark matter components, resulting in a logarithmic profile for the global potential. We tested whether the Schwarzschild method could constrain the characteristic parameters of the system for different sample sizes and whether this was possible without knowledge of the functional form of the potential.

Results. When assuming the true functional form of the potential of the system, the Schwarzschild modelling technique is able to provide an accurate and precise measurement of the characteristic mass parameter of the system and accurately reproduces the light distribution and the stellar kinematics of our mock galaxy. When assuming a different functional form for the potential of the model, such as a flattened Navarro-Frenk-White (NFW) profile, we also constrain the mass and scale radius to their corresponding values. However in both cases, we find that the flattening parameter remains largely unconstrained. This is likely because the information content of the velocity dispersion on the geometric shape of the potential is too small.

Conclusions. Our results using Schwarzschild's method indicate that the mass enclosed can be derived reliably, even if the flattening parameter is unknown, and already for samples containing 2000 line-of-sight radial velocities, such as those currently available. Further applications of the method to more general distribution functions of flattened systems are needed to establish how well the flattening of dSph dark halos can be determined.
\end{abstract}

Key words. dark matter - galaxies: dwarf - galaxies: kinematics and dynamics

\section{Introduction}

In the current cosmological $\Lambda \mathrm{CDM}$ model most of the mass is believed to be in the form of (cold) dark matter. While successful on large scales, on the scales of dwarf galaxies, the model suffers a number of challenges, including the missing satellites problem (Klypin et al. 1999; Moore et al. 1999), the cusp-core conundrum (Hui 2001), and the too-big-to-fail problem (BoylanKolchin et al. 2011), although all may be solved one way or another by considering the effects of baryonic physics (e.g. Zolotov et al. 2012; Brooks et al. 2013; Wetzel et al. 2016; Kim et al. 2018). The dwarf spheroidal satellite galaxies (dSphs or dSph galaxies) of our Milky Way can provide particularly strong constraints on the nature of dark matter, since their high massto-light ratios suggest that they are fully dark-matter dominated (Strigari et al. 2008; Walker et al. 2007; Wolf et al. 2010).

Various methods have been used to develop dynamical models of dSph galaxies using line-of-sight velocity measurements for large samples of individual stars in these systems (e.g. Battaglia et al. 2006, 2008a,b, 2011; Walker et al. 2009a, 2015). Modelling via the Jeans Equations, distribution functions, and orbit superposition methods like Schwarzschild modelling are amongst those most often used (Battaglia et al. 2013). All these methods have in common that they assume that the systems are in dynamical equilibrium.
The Jeans Equations are derived by taking moments of the Collisionless Boltzmann Equation, which itself describes the conservation of probability in phase-space (Binney \& Tremaine 2008). Not every solution of the Jeans Equations has an associated distribution function that is physical (i.e. positive) everywhere. Furthermore, finding a solution requires additional assumptions, for example on the functional form of the density profile and on the velocity anisotropy (because this is generally not known, although see the work by Massari et al. 2018, who determined directly the anisotropy of a sample of stars in the Sculptor dSph using proper motions derived from Gaia and HST). Because Jeans modelling is very flexible and fast, it has become the most widely used tool to model dSph galaxies, particularly in the spherical limit. It has, for example, allowed a robust (independent of the velocity anisotropy) measurement of the mass enclosed within approximately the half light radius of the dSph galaxies (Walker et al. 2009b; Wolf et al. 2010), and the determination that the masses of the classical dSphs are in the range $\sim\left(10^{8}-10^{9}\right) M_{\odot}$ (e.g. Walker et al. 2007). On the other hand, it has not been possible to rule out cusped or cored profiles on the basis of these types of models (e.g. Evans et al. 2009; Strigari et al. 2017).

The Schwarzschild (1979) modelling technique relies on the idea that a system can be seen as a superposition of stellar orbits. In Schwarzschild modelling, one only needs to assume a 
specific gravitational potential form. The method does require a significant amount of computing power and therefore a smaller set of gravitational potentials can be explored in comparison to Jeans modelling. Breddels \& Helmi (2013) applied this method to four dwarf spheroidal galaxies, and by modelling both the second and fourth line-of-sight velocity moments and assuming spherical symmetry they find that, independently of the particular form assumed for the potential, it is possible to constrain not only the mass at around the half-light radius (more precisely at $r_{-3}$ where the logarithmic slope of the luminous density is -3 ) but also the logarithmic slope of the dark matter density.

Most work thus far has assumed that dwarf spheroidal galaxies and their host halos are spherical, despite the fact that their light distribution is typically not spherical (Irwin \& Hatzidimitriou 1995; McConnachie 2012). Furthermore, dark matter halos are predicted to be triaxial (Jing \& Suto 2002) when no baryonic effects are taken into account, although subhalos in cold dark matter simulations that could host $\mathrm{dSphs}$ are only mildly triaxial, and almost axisymmetric (Vera-Ciro et al. 2014). This implies that it is important to establish how many and which of the previously mentioned results still stand when taking into account deviations from spherical symmetry.

Kowalczyk et al. $(2017,2018)$ studied the ability of recovering the mass profile and anisotropy of the remnants of the mergers of dwarf disky galaxies (one postulated channel for the formation of dSphs) when using spherical Schwarzschild models. These latter authors showed that for spherical remnants the method can break the mass-anisotropy degeneracy, whereas for non-spherical (prolate) remnants the anisotropy will always be underestimated, although the total mass profile will be recovered well for data along the minor axis (although not if the data are along the major axis).

On the other hand, Hayashi \& Chiba $(2012,2015)$ used axisymmetric Jeans modelling to infer the axis ratio of the dark matter density distribution $(Q)$ in several $\mathrm{dSphs}$ assuming a constant velocity anisotropy $\beta_{z}$. These latter authors report rather low axis ratios $(Q=[0.3-0.5])$ compared to the observed projected axial ratio in the light $\left(q_{*}^{\prime} \sim 0.7\right)$. These low values are somewhat counterintuitive, though the results may be affected by degeneracies between $Q$, the velocity anisotropy profile, the viewing angle of the $\mathrm{dSph}$, and the inner slope of the dark matter density profile. In Hayashi et al. (2016), a very similar technique was applied to unbinned data, and for the Sculptor dSph for example, the authors found that the flattening parameter is largely unconstrained.

In this work we explore the performance of the Schwarzschild modelling technique in the axisymmetric regime to free ourselves from the assumptions inherent to Jeans models. We test the method on a mock Sculptor-like dSph and consider axisymmetric mass distributions for both the light and the dark matter component and establish how well the characteristic parameters of the potential can be recovered for different sample sizes.

The paper is organised as follows. In Sect. 2, we set up a mock galaxy and simulate a realistic dataset. In Sect. 3 we describe the Schwarzschild method and its implementation in this work. In Sect. 4.1, we then apply the Schwarzschild method and show that we can recover the characteristic mass parameter of the mock galaxy potential, irrespective of the potential flattening parameter assumed. In Sect. 4.2 we model our mock galaxy with an axisymmetric NFW potential form and show that, even in this case, the Schwarzschild method is able to constrain the mass and scale radius to the corresponding values for datasets containing a realistic number of stars. We present our conclusions in Sect. 5 where we also discuss our findings.

\section{The mock galaxy}

\subsection{Potential, luminous density, and characteristic parameters}

We have built a mock galaxy inspired by the Sculptor dSph. We assume a flattened stellar density profile $\left(q_{*}=0.8\right)$, no net rotation, and a line-of-sight velocity dispersion of $\sim 10 \mathrm{~km} \mathrm{~s}^{-1}$ (Mateo 1998; Battaglia et al. 2008a; Walker et al. 2009b). For simplicity, we have set up the mock galaxy following Evans (1993), who uses an elementary distribution function to describe a composite axisymmetric system. This distribution function is ergodic, meaning that it leads to a velocity ellipsoid that is isotropic and has a constant amplitude and thus is not generic ${ }^{1}$. Even though the velocity anisotropies of most classical dSphs do not appear to be too far from isotropic (see e.g. Hayashi \& Chiba 2015; Hayashi et al. 2016; Hayashi \& Obata 2019; Massari et al. 2019, but also Massari et al. 2018), our specific choice of an isotropic model might be a simplification of reality.

The gravitational potential of the composite system as a whole follows the form

$\Phi_{\mathrm{E}}(R, z)=\frac{1}{2} v_{0}^{2} \ln \left(R_{\mathrm{c}}^{2}+R^{2}+\frac{z^{2}}{q^{2}}\right)+\Phi_{0}$,

where $(R, \phi, z)$ denote the cylindrical coordinates. Here, $v_{0}^{2}$ relates to the mass of the system and $R_{\mathrm{c}}$ is the core radius. The parameter $q$ is the axial ratio of the potential, and has to satisfy $1 / \sqrt{2}=0.707 \leq q \leq 1.08$ where the lower limit is set by the condition that the spatial density is positive everywhere (Binney $\&$ Tremaine 2008) and the upper limit yields a composite distribution function of the form used by Evans (1993) that is positive everywhere. The zero point of the potential is set by $\Phi_{0}$.

The density profile of the stellar component is described by

$\rho_{\text {lum }}(R, z)=\frac{\rho_{0} R_{\mathrm{c}}^{p}}{\left(R_{\mathrm{c}}^{2}+R^{2}+z^{2} / q_{*}^{2}\right)^{p / 2}}$,

where $\rho_{0}$ is the central density, $p$ denotes a slope parameter, and $q_{*}$ is the axial ratio of the stellar density. The associated stellar distribution function is given by

$f_{\text {lum }}(E) \propto \exp \left[-p E / v_{0}^{2}\right]=\exp \left[-p \Phi_{\mathrm{E}} / v_{0}^{2}\right] \exp \left[-p v^{2} / 2 v_{0}^{2}\right]$,

where $E$ is the sum of the gravitational potential and kinetic energies of a star.

Throughout the paper we define the flattening of a system as the axial ratio of the equipotential contours. In the Evans model, $q_{*}=q$ and therefore the axial ratio of the stellar density component is the same as that of the potential (not the density) of the composite system. For $q<1$, the system is said to be oblate, and prolate for $q>1$. The surface brightness profile of the mock galaxy can be found by integrating the luminous density along the line-of-sight.

The line-of-sight velocity profile is exactly Gaussian with a velocity dispersion that is isotropic and constant everywhere:

$\sigma_{\mathrm{E}}=\frac{v_{0}}{\sqrt{p}}$

1 Nor is this distribution function ideal as we see below, because it provides very little information on the symmetries of the system. 


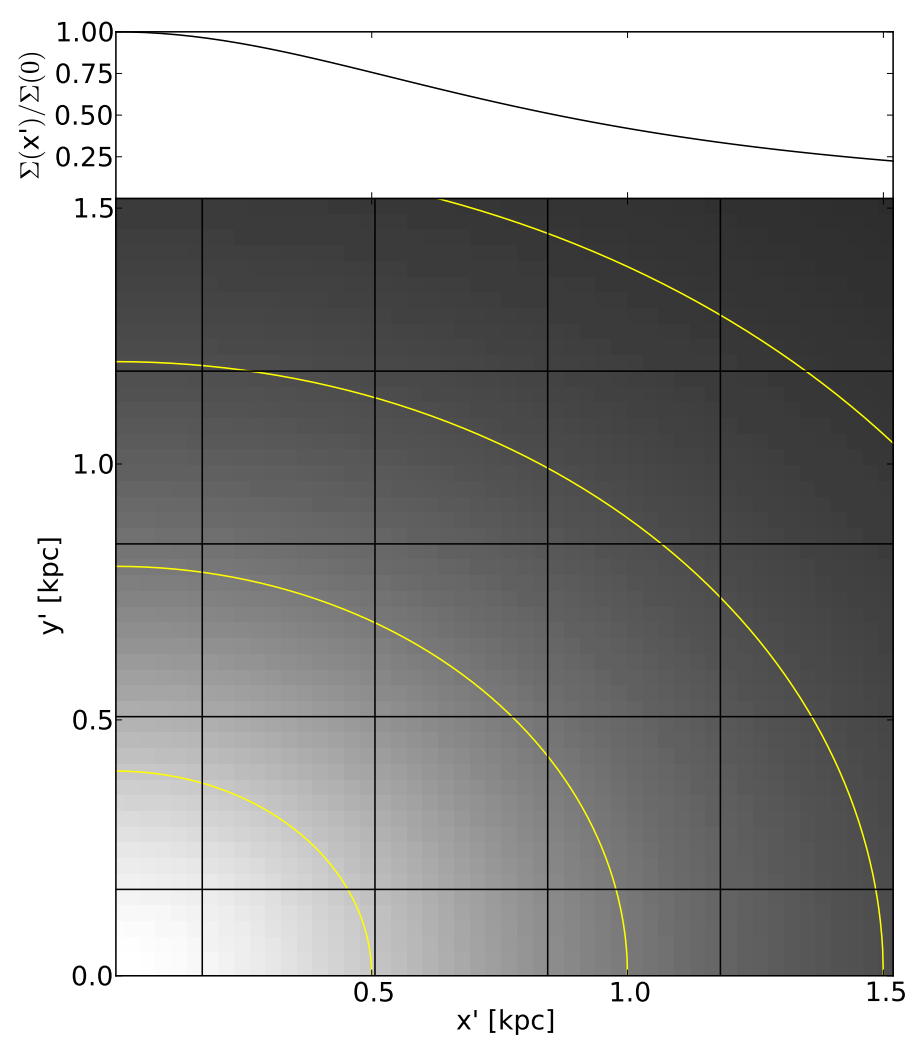

Fig. 1. Surface brightness profile of our mock galaxy in an edge-on view. The black horizontal and vertical lines show the boundaries of the kinematic bins. We only show the positive quadrant of our FOV $\left(x^{\prime}>\right.$ $\left.0 \mathrm{kpc}, y^{\prime}>0 \mathrm{kpc}\right)$. The yellow contours correspond to the isophotes of the system $\left(q_{*}=q=0.8\right)$. In the top panel we have plotted the surface brightness normalised to its central value as function of $x^{\prime}$, i.e. along the (projected) major axis of the galaxy.

and independent of the inclination, scale radius, and flattening parameter.

We choose here $v_{0}=20 \mathrm{~km} \mathrm{~s}^{-1}, R_{\mathrm{c}}=1 \mathrm{kpc}, q=0.8$, and $p=3.5$ for our mock galaxy. These values result in a velocity dispersion of roughly $10.7 \mathrm{~km} \mathrm{~s}^{-1}$. For these values of $p$ and $q$, the central total density should be at least 1.13 times the central stellar density to yield positive phase-space densities for both the stellar and dark components everywhere.

\subsection{Observing the mock galaxy}

We place the mock galaxy at a distance of $80 \mathrm{kpc}$, and "observe" it with a square field of view (FOV), centred on the mock galaxy, with a size of $7832^{\prime \prime} \times 7832^{\prime \prime}$ (which then corresponds to roughly $3 \times 3 \mathrm{kpc})^{2}$. The sky coordinates therefore range from roughly $-1.5 \mathrm{kpc}$ to $+1.5 \mathrm{kpc}$. Throughout this work we assume an edgeon view.

In Fig. 1 we show the resulting 2D surface brightness profile of the mock galaxy for an edge-on view and as a function of the sky coordinates $x^{\prime}$ and $y^{\prime}$. Since the galaxy is axisymmetric, we only show the positive quadrant. Contours of constant surface brightness follow ellipses with axial ratios $q_{*}^{\prime}$, which because of the edge-on view are identical to those of the intrinsic density (i.e. $q_{*}^{\prime}=q_{*}=0.8$ ). The $1 \mathrm{D}$ surface brightness profile along the

\footnotetext{
2 For an extended system like the one studied here, it would be better to use a larger FOV, or to have multiple pointings. The size of the FOV used here is however reasonable for $\mathrm{dSph}$ galaxies like Sculptor.
}

major axis is plotted in the top panel of this figure. The surface brightness decreases by a factor of two with respect to its central value at a projected ellipsoidal radius of $0.86 \mathrm{kpc}$, however the projected half light radius is much larger $(3.87 \mathrm{kpc})$.

We generate kinematic data for the mock galaxy by drawing positions of stars following the luminous density distribution (see Eq. (2)) and velocities from the Gaussian distribution function (see Eq. (3)). We thus assume that the dataset of the stars with measured line-of-sight velocities follows the same distribution as the light.

The typical line-of-sight velocity measurements of individual stars have errors of order $\mathrm{d} v=2 \mathrm{~km} \mathrm{~s}^{-1}$ (Battaglia et al. 2008b; Walker et al. 2009a). Therefore, to simulate a realistic kinematic dataset we convolve the line-of-sight velocities with a Gaussian distribution having a standard deviation of $2 \mathrm{~km} \mathrm{~s}^{-1}$. We compute velocity moments by combining the velocities of all available stars in a certain spatial bin on the sky (in what follows a kinematic bin) in our FOV. This binning is thus performed in the projected sky coordinates $x^{\prime}$ and $y^{\prime}$. The black horizontal and vertical lines visible in Fig. 1 show the borders of the kinematic bins from the positive quadrant. The velocity moments are estimated by correcting for the measurement errors (see Appendix A), similarly to Breddels et al. (2013). We assume that the surface brightness profile can be measured without error in much smaller spatial bins on the sky (which we refer to as light bins). To produce a reasonable galaxy, we also assume that the 3D light distribution is known to much larger radii, but for many fewer bins (more details can be found in Sect. 3.1).

\section{The Schwarzschild orbit superposition method}

In Schwarzschild modelling, orbits are used as building blocks of a dynamical system. Given a potential $\Phi$, a complete set of orbits are integrated numerically and for each orbit the predicted observables are stored in a so-called orbit library. Varying the parameters of the potential (or varying the potential form as a whole), results in different libraries. The library that provides a combination of weighted orbits that best matches the observations (light profile + kinematics) is said to yield the best-fit parameters of the potential. The orbital weights themselves provide the corresponding distribution function. Since the orbital weights are positive by construction, the distribution function is non-negative everywhere.

\subsection{Generating orbit libraries}

In this paper we use a slightly modified version of the Schwarzschild code from van den Bosch et al. (2008), who modelled the elliptical galaxy NGC 4365. In what follows, we briefly describe how we generated the orbit libraries, how the orbital integration has been done, and how the libraries are stored. For more information we refer the reader to van den Bosch et al. $(2008)^{3}$.

Given an energy $E_{i}$, initial positions $x_{0}$ and $z_{0}$ are sampled on an open polar grid, which is defined by $N_{I_{2}}$ polar angles and $N_{I_{3}}$ radii in between a thin orbit and the equipotential. The polar

\footnotetext{
3 We note that the Schwarzschild code by van den Bosch et al. (2008) was developed to model triaxial systems, and therefore also generates initial conditions for box orbits, which have zero time-averaged angular momentum and which can cross the centre (Schwarzschild 1979, 1993). In an axisymmetric potential $L_{z}$ is conserved and such box orbits will therefore never attain velocities in the azimuthal direction. As this could cause non-axisymmetries in our model we do not specifically generate box orbits.
} 
angles are sampled linearly, but to obtain a better sampling of orbits near the major axis of the system, $50 \%$ of the polar angles are sampled from the $z$-axis towards $10^{\circ}$ above the midplane, and the remaining $50 \%$ from $10^{\circ}$ down to the $z=0$ plane. The initial $y$-coordinates and initial velocities in the $x$ - and $z$-directions are set to zero. The initial velocities in the $y$-direction, $v_{y, 0}$, are determined by $E_{i}-\Phi\left(x_{0}, 0, z_{0}\right)=0.5 v_{y, 0}^{2}$. This is done for all $N_{\text {ener }}$ energies, which are defined by $E_{i}=\Phi\left(x=x_{i}, y=0, z=0\right)$. The locations $x_{i}$ that fix the energy grid are logarithmically sampled between $25 \mathrm{pc}$ and $50 \mathrm{kpc}$ from the centre. Such sampling also makes it possible to model a reasonable light distribution at distances well outside our FOV (more detailed information about the $3 \mathrm{D}$ grid used for this purpose is given below). This "orbit library" therefore consists of $N_{\text {orb }}=N_{\text {ener }} \times N_{I_{2}} \times N_{I_{3}}$ orbits ( $z$ tubes in our axisymmetric potential).

To account for slowly precessing orbits in the library we also compute 17 copies of each orbit, where each copied orbit is subsequently rotated by $10^{\circ}$ in the $x y$-plane. These 18 copies are summed into a single orbit and replace the non-rotated orbit, such that each orbit now follows the axisymmetric requirements. Besides ensuring the axisymmetric behaviour of our models, adding rotations also increases the sampling of an orbit. We also note that each orbit has a counter-rotating sibling, obtained by appropriately changing the sign of the velocity vector.

We further improve the accuracy of the model by "dithering": every orbit is split into $N_{\text {dither }}^{3}$ suborbits by replacing each of its three nonzero initial coordinates by $N_{\text {dither }}$ slightly different coordinates. The initial conditions of all suborbits are found by increasing $N_{\text {ener }}, N_{I_{2}}$, and $N_{I_{3}}$ by a factor of $N_{\text {dither. The observ- }}$ ables of each set of adjacent $N_{\text {dither }}^{3}$ suborbits are combined and stored as being the observables of the (bundled) orbit. Choosing an odd number for $N_{\text {dither }}$ ensures that the original orbit is the central suborbit of the bundle. In all our Schwarzschild models we use $N_{\text {dither }}=5$. Every main orbit is thus made from a bundle of $5^{3}=125$ neighbouring suborbits.

We use a Runge Kutta integrator to compute the stellar trajectories over roughly 200 orbital timescales. We require that the energy of each suborbit is always conserved better than $1 \%$ by increasing the accuracy of the integrator if necessary. For each orbit the kinematic information is stored in a velocity grid, which consists of a line-of-sight velocity axis ( $N_{v}$ velocity bins) and an axis associated to the location on the sky ( $N_{\text {kin }}$ kinematic bins). On equally spaced time intervals, a count is added to the element of the grid associated to the velocity and location at the given time. The sky projected path of the orbit is determined in a similar way and stored in the surface brightness grid con-

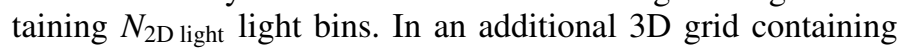
$N_{3 \text { D light }}=800$ bins (40 radial, 5 azimuthal, and 4 polar bins in the positive octant) the $3 \mathrm{D}$ path of an orbit is stored. This 3D grid contains the same radii as used to sample the energies for the initial conditions and thus reaches well beyond the FOV (in contrast to the velocity and surface brightness grid). It is used to match the (3D) light distribution of the system at such radii.

In this work we set $N_{2 D}$ light equal to $99 \times 99=9801$ and $N_{\text {kin }}$ to $9 \times 9=81$, unless stated otherwise. The velocity axis of the velocity grid contains $N_{v}=41$ bins and has a total velocity width of $80 \mathrm{~km} \mathrm{~s}^{-1}$, such that we cover velocities up to $\pm 4 \sigma_{\mathrm{E}}$. The central velocity bin is centred on $0 \mathrm{~km} \mathrm{~s}^{-1}$. To be able to track how long an orbit spends in a given kinematic bin, we need to account for the time spent with velocities outside the velocity grid (at that position). Therefore counts are also added to the first or last velocity bin if velocities are beyond the limits of the velocity grid.
When taking too few (i.e. too wide) velocity bins for the velocity grid, the velocity moments might not be recovered correctly. We checked that if we bin the theoretical Gaussian line-of-sight velocity profile of our mock galaxy as described above, thus discarding the contribution of velocities that are outside the range of the grid, the velocity moments are recovered well, that is the first and third moments are not affected, while the second and fourth velocity moments might result in relative errors of order $0.1 \%$ and $2 \%$ given the choices made for binning the velocity data.

\subsection{Fitting orbital weights}

Once the orbit libraries are in place, we find the orbital weights such that the total luminous mass, the surface brightness profile and the kinematics within the FOV, and the 3D light profile of the system are reproduced.

The 2D light profile is fitted using the surface brightness grid, where we define:

$m_{j}^{\text {light }}=\sum_{i=1}^{N_{\text {orb }}} w_{i} m_{i j}^{\text {light }}$,

where we sum over all orbits $i$. Here, $m_{i j}^{\text {light }}$ is the fraction of time orbit $i$ spent in light bin $j$ and $m_{j}^{\text {light }}$ is the fractional surface brightness in light bin $j$. The orbital weights are denoted by $w_{i}$ and add to unity by construction. The 3D light profile is fitted similarly using the 3D grid.

At the same time as we fit the light, we also fit the kinematics. In every kinematic bin $k$ we compute the first four massweighted velocity moments $\left\langle v_{k}^{n}\right\rangle$ by defining:

$m_{k}^{\mathrm{kin}}\left\langle v_{k}^{n}\right\rangle=\sum_{i=1}^{N_{\text {orb }}} w_{i} m_{i k}^{\mathrm{kin}}\left\langle v_{i k}^{n}\right\rangle$,

where again we sum over all orbits $i$. This time $m_{i k}^{\text {kin }}$ is the fraction of time orbit $i$ spent in kinematic bin $k$ and $m_{k}^{\text {kin }}$ is the fractional surface brightness in kinematic bin $k$. The $n$th moment of orbit $i$ in kinematic bin $k$ is given by $\left\langle v_{i k}^{n}\right\rangle$ :

$\left\langle v_{i k}^{n}\right\rangle=\frac{\sum_{l=2}^{N_{v}-1} h_{i k l} v_{\mathrm{cen}, l}^{n} \Delta v}{\sum_{l=2}^{N_{v}-1} h_{i k l} \Delta v}$,

where $\Delta v$ is the size of the velocity bin and $h_{i k l}$ is the fraction of time that orbit $i$ spent in kinematic bin $k$ and velocity bin $l$. Velocity bin $l$ has velocity range $\left[v_{\text {cen, } l}-\frac{1}{2} \Delta v, v_{\text {cen }, l}+\frac{1}{2} \Delta v\right]$, where $v_{\text {cen }, l}$ denotes its central velocity. We sum over the $N_{v}$ velocity bins, although we discard the contributions of the first and last velocity bin. This is done since we did not set a stringent outer velocity boundary in these velocity bins: as described before, counts will be added here even if a star has a velocity outside the range of the grid and therefore the typical velocities of these bins are not known. We note that $m_{i k}^{\mathrm{kin}}=\sum_{l=1}^{N_{v}} h_{i k l}$ with this choice.

Now that we have defined the relation between the observables and the quantities in our model, we can describe how we fit the orbital weights. The fit is based on minimising $\chi_{\text {tot }}^{2}$ :

$\chi_{\text {tot }}^{2}=\sum_{u=1}^{N_{\text {obs }}}\left[\frac{\operatorname{Model}[u]-\operatorname{Data}[u]}{\operatorname{Error}[u]}\right]^{2}$, 
where $u$ runs over all $N_{\text {obs }}$ observables. The number of observables is given by:

$N_{\text {obs }}=1+N_{2 \mathrm{D} \text { light }}+N_{3 \mathrm{D} \text { light }}+4 N_{\text {kin }}$,

which includes the contribution of the total light of the system, the fractional light for each 2D and 3D light bin, and the four velocity moments for each kinematic bin, respectively. We choose to use four velocity moments, since using higherorder moments might reduce the degeneracy between the velocity anisotropy and the mass profile (e.g. Merrifield \& Kent 1990; Richardson \& Fairbairn 2013). We do not use higher moments since these are observationally harder to constrain. The odd velocity moments are used to ensure that the Schwarzschild model also matches the observed mean rotation and the skewness of velocity distribution.

We use a non-negative least-squares solver to ensure that all orbital weights are positive. The light is weighted by assigning an error of $2 \%$ to each of the 2D and 3D light bins. We note that we can investigate the individual contribution to the total $\chi_{\text {tot }}^{2}$ by decomposing it; for example,

$\chi_{\text {tot }}^{2}=\chi_{\text {total light }}^{2}+\chi_{2 \mathrm{D} \text { light }}^{2}+\chi_{3 \mathrm{D} \text { light }}^{2}+\chi_{\text {kin }}^{2}$.

We stress that $\chi_{\text {tot }}^{2}$ is being minimised. We do not minimise the terms on the right-hand side individually. The term associated to the total light of the system turns out to be negligible, since it is always recovered very well. The same holds for $\chi_{3 \mathrm{D} \text { light }}^{2}$. These terms are only added to Eq. (8) to ensure that the model returns a realistic galaxy (in the sense that the luminous component might resemble a galaxy). Most of the constraining power therefore comes from the surface brightness profile and the kinematics.

\section{Results}

In this section we show that the Schwarzschild method can recover some of the characteristic parameters of the mock Sculptor-like dwarf spheroidal galaxy. We first show in Sect. 4.1 that if the true functional form of the potential of the system is known, we can constrain the characteristic mass parameter of the mock galaxy. In reality however, the true functional form of the potential of the system is not known. Therefore, in Sect. 4.2 we demonstrate how well we can constrain characteristic parameters when assuming an axisymmetric form of a Navarro-FrenkWhite (NFW, Navarro et al. 1996) potential.

\subsection{Two parameter Evans models: recovering the mock galaxy parameters}

Here we assume the true functional form of the potential of the system is known, and we use it to build the orbit libraries for the Schwarzschild models. Our aim is to establish whether we can recover the correct values of the characteristic input parameters with this method. To this end we make a grid of models in which we vary the values of the characteristic parameters $q$ and $v_{0}$ (see Eq. (1)). We fix the core radius to $R_{\mathrm{c}}=1 \mathrm{kpc}$, that is, to its true value. We sample $q$ from 0.72 to 0.96 , and $v_{0}$ from $11 \mathrm{~km} \mathrm{~s}^{-1}$ to $29 \mathrm{~km} \mathrm{~s}^{-1}$, with higher sampling (decided iteratively) with steps in $q$ of 0.02 and in $v_{0}$ of $1 \mathrm{~km} \mathrm{~s}^{-1}$. We name the models by the values of their parameters: qXXvYY in which XX $=100 q$ and $\mathrm{YY}=v_{0}$ in $\mathrm{km} \mathrm{s}^{-1}$. For the orbit sampling, we set $N_{\text {ener }}=32$, $N_{I_{2}}=32$ and $N_{I_{3}}=16$ such that a total of $32 \times 32 \times 16 \times 5^{3}=$ 2048000 suborbits are integrated (see Sect. 3.1) and $2 \times 32 \times$ $32 \times 16=32768$ orbital weights are determined (see Sect. 3.2).

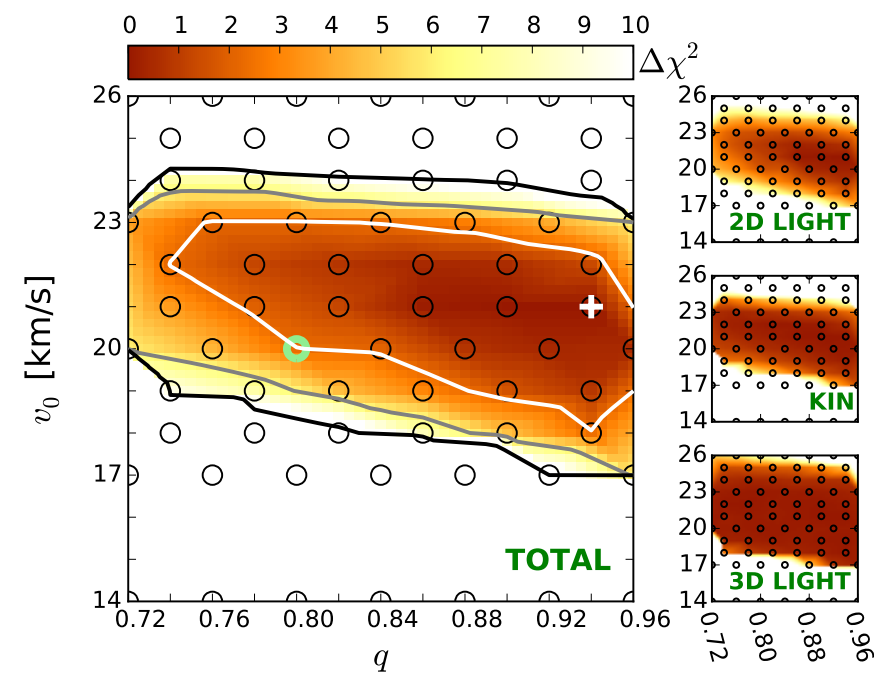

Fig. 2. $\Delta \chi^{2}$-distribution of the characteristic parameters $q$ and $v_{0}$ of the Evans models obtained after applying the Schwarzschild method. In this case our mock kinematic data consist of $10^{5}$ stars inside the FOV $(3 \times$ $3 \mathrm{kpc}$ ). We use $9 \times 9$ kinematic bins and assume the functional form of the potential of the system and inclination are known. The black circles show the locations where the Schwarzschild models were evaluated. The green circle indicates the input parameters of the mock system. The best-fit model is indicated by the white cross and recovers the mock galaxy mass parameter. In white, grey, and black we show the $\Delta \chi^{2}=$ $[2.3,6.18,11.8]$-contours, respectively. The coloured landscape on the left shows interpolated $\Delta \chi^{2}$-values, and goes up to a maximum of $\Delta \chi^{2}=$ 10 . On the right we show the $\Delta \chi^{2}$-landscapes when decomposing the landscape into $\chi_{2 \mathrm{D} \text { light }}^{2}$ (top), $\chi_{\text {kin }}^{2}$ (middle), or $\chi_{3 \mathrm{D} \text { light }}^{2}$ (bottom). These right panels sum up to the large panel. We stress that only $\chi_{\text {tot }}^{2}$ is being minimised in the fit.

\subsubsection{Results for a large sample}

We start with an idealised case in which the kinematic data consist of $10^{5}$ stars. For $9 \times 9$ kinematic-bins on the sky, the typical error of the velocity dispersion in a kinematic bin is $\sim 0.25 \mathrm{~km} \mathrm{~s}^{-1}$

The large panel of Fig. 2 shows the results obtained by fitting the Schwarzschild models to the data. The small black circles show the grid of tested values for $q$ and $v_{0}$, the green circle the true input values, and the white cross indicates the values of the parameters corresponding to the maximum likelihood estimator (MLE). For the best-fit model q94v21 we find $\chi_{\text {tot }}^{2}=207.7$. The contribution of the kinematics (see Eq. (8)) to this value is 205.6. Using 81 kinematic bins to fit four velocity moments, this corresponds to 0.64 per kinematic constraint.

We computed $\Delta \chi^{2}\left(q, v_{0}\right)=\chi_{\text {tot }}^{2}\left(q, v_{0}\right)-\min \left[\chi_{\text {tot }}^{2}\right]$ for each of these models and define $68 \%, 95 \%$, and $99.7 \%$ confidence intervals (white, grey, and black contours, respectively) at $\Delta \chi^{2}=$ $[2.3,6.18,11.8]$ (Press et al. 1992) ${ }^{4}$. The coloured background shows the $\Delta \chi^{2}$ landscape and is truncated at $\Delta \chi^{2}=10$. The smaller panels on the right show the $\Delta \chi^{2}$ landscapes when only considering $\chi_{2 \mathrm{D} \text { light }}^{2}$ (top), $\chi_{\text {kin }}^{2}$ (middle), or $\chi_{3 \mathrm{D} \text { light }}^{2}$ (bottom). The $\Delta \chi^{2}$ landscape based on $\chi_{\text {tot }}^{2}$ is therefore slightly dominated by the differences in $\chi_{2 \mathrm{D} \text { light }}^{2}$, although the kinematics provide similar constraints

To estimate the error on the mass parameter we first marginalise over the flattening parameter by selecting for each

4 We used the scipy.interpolate.LinearNDInterpolator to interpolate the $\Delta \chi^{2}$. Although using linear interpolation might not be optimal, it does not strongly affect the quantitative results of the paper. 


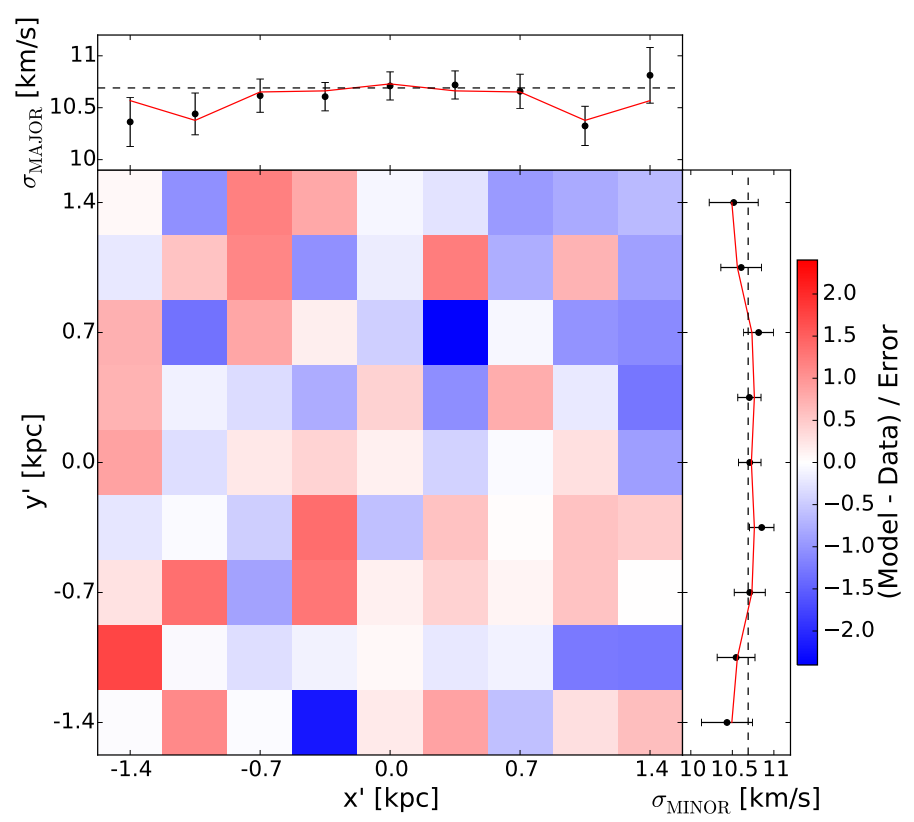

Fig. 3. Difference between the best-fit and the observed velocity dispersion in terms of the observed error, for all $9 \times 9$ kinematic bins The figure is obtained after fitting the q94v21 library to our mock data consisting of $10^{5}$ stars with measured line-of-sight velocities in our FOV, assuming an edge-on view. Top and right panels: fit (red full line) obtained along the major and minor axis, respectively. The data points with $68 \%$ error bars are shown in black. Black dashed lines indicate the true velocity dispersions from theory (Eq. (4)).

$v_{0}$ the minimum $\Delta \chi^{2}$ along $q$. We define the $68 \%$ error at those values where $\Delta \chi^{2}=1.0$ (Press et al. 1992). For this experiment we find $v_{0}=21_{-2.11}^{+1.33} \mathrm{~km} \mathrm{~s}^{-1}$. We therefore conclude that we can recover the input mass parameter of our mock galaxy well, but as Fig. 2 shows we do not tightly constrain the flattening parameter $q$.

In Fig. 3 we show how well the velocity dispersion is fitted in the best-fit q94v21 model. For each kinematic bin, we show how much the model deviates from the data expressed in units of the error on the data. The top panel shows the fit along the major axis while the panel on the right shows the fit along the minor axis. The axisymmetric behaviour of our models can be clearly seen in the panels. The observed velocity moments are subject to sampling effects and measurement errors and thus do not exactly obey such symmetry conditions. The figure shows that the fit is very good (and is almost indistinguishable from the fit obtained for what would be the input parameters model, i.e. q80v20).

\subsubsection{Downsampling and folding data}

We now consider the more realistic case of a sample of $10^{4}$ stars with measured line-of-sight velocities. We do not change our assumption that the surface brightness profile can be measured without error in our light bins, and therefore here we only investigate the influence of the number of stars with line-ofsight velocities available. To reduce the observed uncertainties on the kinematics we decided to fold the kinematic data (but not the light). Since the system is axisymmetric, we fold our data into the kinematic bins located in the first quadrant. We can simply move each star towards its corresponding kinematic bin without changing its velocity, because our system has an identical Gaussian line-of-sight profile everywhere (see Sect. 2.1). In
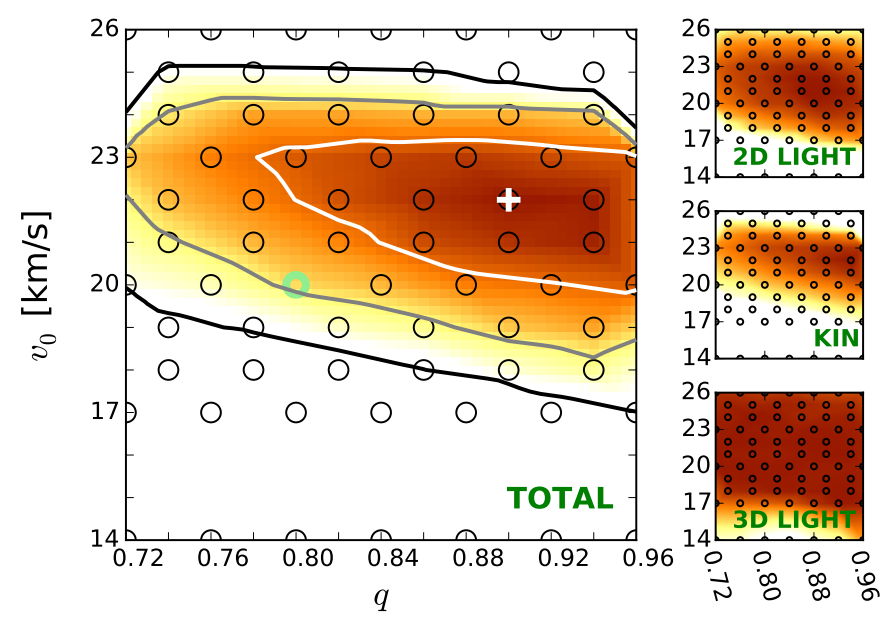

Fig. 4. Similar to Fig. 2, but now using $10^{4}$ stars for the kinematics and using the approach of folding the data from $9 \times 9$ into $5 \times 5$ kinematic bins. The parameter inferences are similar, though slightly larger masses are preferred.

general, however, one should change the velocities following the assumed symmetry.

Since we fold the kinematic data from $9 \times 9$ bins of our FOV into the first quadrant, we effectively have $10^{4}$ stars with measured line-of-sight velocities located in the resulting $5 \times 5$ kinematic bins. A typical kinematic bin now contains 400 stars with measured line-of-sight velocities on average, and the typical error on the velocity dispersion is $\sim 0.45 \mathrm{~km} \mathrm{~s}^{-1}$.

We fit the folded data with the Schwarzschild orbit superposition method and find the MLE for model q90v22 (see Fig. 4). As in the case of $10^{5}$ stars, and thus as expected, the flattening parameter remains fairly unconstrained. We find a slightly larger mass parameter $v_{0}=22_{-1.44}^{+1.02} \mathrm{~km} \mathrm{~s}^{-1}$, but $v_{0}=20 \mathrm{~km} \mathrm{~s}^{-1}$ is still within the $95 \%$ confidence region.

For the best-fit model q90v22 we find $\chi_{\text {tot }}^{2}=16.5$. The contribution of the kinematics (see Eq. (8)) to this value is 13.2. Both values are much lower than in the case of $10^{5}$ stars, and this can be explained by the decrease in the number of kinematic constraints and the fact that the data have now been folded.

It is encouraging that a more realistic number of stars with measured line-of-sight velocities still gives such tight constraints. Comparing the folded case with $10^{4}$ stars to the nonfolded case with $10^{5}$ stars, the 2D $68 \%$-probability contours are shifted towards just slightly larger masses. We note that the uncertainty on the mass parameter did not increase.

To further test how the results depend on the number of stars with measured line-of-sight velocities, we even further decreased this number of stars to a sample of 2000 stars. This is the typical size of currently available kinematic datasets used to put constraints on the mass of dSph galaxies (e.g. Walker et al. 2009b; Breddels \& Helmi 2013; Hayashi \& Chiba 2015). We again fold the data from $9 \times 9$ into $5 \times 5$ kinematic bins. The resulting typical error on the velocity dispersion in a kinematic bin is then $\sim 0.9 \mathrm{~km} \mathrm{~s}^{-1}$. In this case we find a best-fit model q92v23 $\left(\chi_{\text {tot }}^{2}=38.9, \chi_{\text {kin }}^{2}=32.6\right)$. The $\Delta \chi^{2}$ distribution is shown in Fig. 5. The best models are again the most spherical ones, although statistically the flattening parameter remains unconstrained. The region spanned by the contour drawn at $\Delta \chi^{2}=11.8$ is of similar size, but is shifted towards slightly higher masses $\left(\Delta v_{0} \sim 1 \mathrm{~km} \mathrm{~s}^{-1}\right)$ in comparison to the case of $10^{4}$ stars. The true q80v20 model is nevertheless still within the inferred $99.7 \%$ confidence interval. 

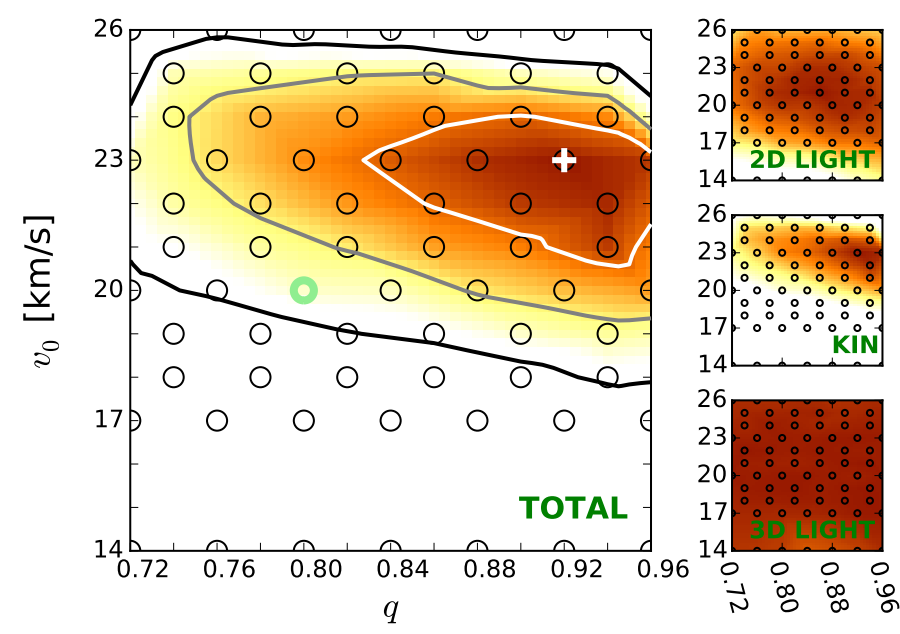

Fig. 5. Similar to Fig. 4, now using 2000 stars for the kinematic data The parameter inferences are similar, though slightly larger masses are inferred $\left(\Delta v_{0} \sim 1 \mathrm{~km} \mathrm{~s}^{-1}\right)$.

At first sight it may seem unrealistic that the case of 2000 results in slightly tighter constraints on the parameters of interest. We however note that the differences are minor and that these might be related to the fact that only one data realisation is studied for each case. We also note that not only are the parameters of the potential being fitted in Schwarzschild models but also the orbital weights. This implies that the fitted distribution function can therefore be quite different from one model to the next (especially without the use of regularisation), and the marginalisation over the orbital weights is not being considered when computing the confidence contours on the potential parameters shown in Fig. 5 (also see Magorrian 2006). Nonetheless, when comparing the predicted line-of-sight velocity dispersions for the range of models within these contours (see Fig. 12 in Sect. 4.2.2) weaker constraints are in fact obtained for smaller datasets, as expected.

The weak trend found for smaller samples to result in slightly higher values of $v_{0}$ may be due to the fact that, for small radii (compared to $R_{\mathrm{c}}$ ), the potential (see Eq. (1)) is proportional to $v_{0}^{2}\left[\ln R_{\mathrm{c}}^{2}+\left(R / R_{\mathrm{c}}\right)^{2}\right]+\left(v_{0} / q\right)^{2}\left(z / R_{\mathrm{c}}\right)^{2}$. Therefore, there is a weak degeneracy in the term $v_{0} / q$, that may manifest itself more when the sampling is sparse, and thus lead to a small shift in preferred values of $v_{0}$ for larger $q$.

From the tests performed in this section we conclude that, with a kinematic sampling that follows the light, we cannot aim to constrain the flattening parameter of an isothermal dSph galaxy ${ }^{5}$, not even if the true functional form of the potential of the system is known. This is likely because the information content in a velocity dispersion regarding the geometric shape of the potential is too small (since $\sigma$ is constant across the whole system). We can however still reliably constrain the mass parameter of such a system, that is, even though the true flattening parameter remains unknown. This can already be done for a realistic number of stars.

\subsection{Axisymmetric NFW models}

We have shown that the Schwarzschild method can correctly constrain the mass parameter when the true functional form of the potential of the system is known. Now, we tackle the problem more realistically by allowing a different functional form for the

\footnotetext{
5 Slightly better results can be obtained by sampling uniformly with distance, see Appendix B.
}

potential of the model. We consider an axisymmetric NFW profile, and follow the parametrisation of Vogelsberger et al. (2008):

$\Phi_{\mathrm{V}}(\tilde{r})=-4 \pi G \rho_{0} R_{\mathrm{s}}^{3}\left[\frac{\ln \left(1+\tilde{r} / R_{\mathrm{s}}\right)}{\tilde{r}}\right]$,

where $R_{\mathrm{S}}$ is the scale radius and $\rho_{0}$ a characteristic density parameter. In comparison to the spherical NFW profile, the radius $r=\sqrt{R^{2}+z^{2}}$ is replaced by a newly defined radius:

$\tilde{r}=\frac{\left(r_{a}+r\right) r_{E}}{r_{a}+r_{E}}$

where, for the axisymmetric case, $r_{E}=\sqrt{\left(\frac{R}{a}\right)^{2}+\left(\frac{z}{c}\right)^{2}}$ is the ellipsoidal radius with $a$ and $c$ specifying the relative lengths of the major and minor axes, and where $r_{a}$ is a transition radius. In addition, we require that $2 a^{2}+c^{2}=3$, such that when $a=c=1$, this results in the spherical NFW profile. For $r \gg r_{a}, \tilde{r} \rightarrow r$, whereas for $r \ll r_{a}, \tilde{r} \rightarrow r_{E}$. Therefore, the gravitational potential is axisymmetric in the central regions and becomes spherical in the outer regions. We set the transition radius to $r_{a}=10 \mathrm{kpc}$. In all our Vogelsberger models we keep the transition radius $r_{a}$ fixed.

To additionally guarantee that the total mass density is positive up to at least the orbits possessing the highest energies in our library $(\sim 50 \mathrm{kpc})$, the flattening parameter must satisfy $c / a \gtrsim 0.7$ for a case with $R_{\mathrm{s}}=1 \mathrm{kpc}$. For smaller scale radii, increased lower-limit values of $c / a$ are needed to satisfy the positive density criterion.

For convenience, we define a characteristic mass parameter, $M_{1 \mathrm{kpc}}$ expressed in units of $M_{\odot}$, which corresponds to the total enclosed mass within $1 \mathrm{kpc}$ from the centre for a spherical NFW profile with scale radius $R_{\mathrm{s}}$, that is,

$$
M_{\mathrm{NFW}}\left(r=1 \mathrm{kpc} \mid R_{\mathrm{S}}\right)=4 \pi \rho_{0} R_{\mathrm{s}}{ }^{3}\left[\ln \left(\frac{R_{\mathrm{s}}+r}{R_{\mathrm{S}}}\right)-\left(\frac{r}{R_{\mathrm{S}}+r}\right)\right]_{1 \mathrm{kpc}} .
$$

From this equation we determine the value of $\rho_{0}$, and it is this value of $\rho_{0}$ that we use for the axisymmetric Volgelsberger potential in Eq. (11).

\subsubsection{The "matched" Vogelsberger model}

Before we can test the Schwarzschild orbit superposition method while assuming Vogelsberger mass models, we need to know when a result can be considered satisfactory. Since we could not constrain the flattening parameter for the case when the true functional form of the potential of the system is known, we do not aim to constrain the flattening parameter for the Vogelsberger models. Nevertheless, the corresponding scale radius $R_{\mathrm{s}}$ and mass $M_{1 \mathrm{kpc}}$ of our system depend on the $c / a$-value assumed. In this section we therefore establish what are good parameters for the mass $M_{1 \mathrm{kpc}}$, scale radius $R_{\mathrm{s}}$, and flattening parameter $c / a$, such that the properties of the Evans mock galaxy are best reproduced.

Because most stars of our mock galaxy will have projected radii in between 0.5 and $2.0 \mathrm{kpc}$ from the centre, we require that the flattening of the "matched" Vogelsberger model should be comparable to that of the mock galaxy over this region. At a given position we define the Vogelsberger potential flattening $q_{\mathrm{V}}$ as the axis ratio of the equipotential contour that goes through that point. For a position $(R, z)$, we therefore define $q_{\mathrm{V}}(R, z)=z_{\Phi} / R_{\Phi}$, where $\Phi\left(R=0, z_{\Phi}\right) \equiv \Phi\left(R_{\Phi}, z=0\right) \equiv \Phi(R, z)$. 

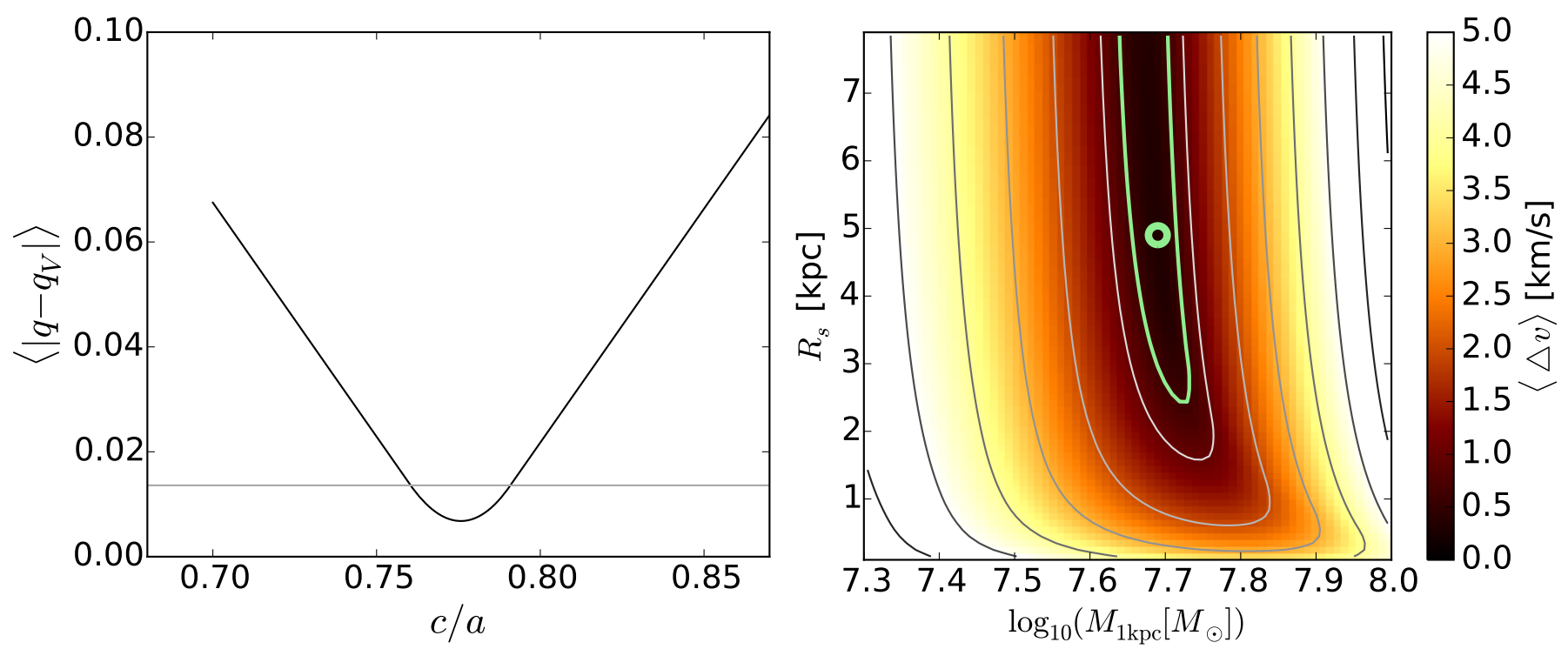

Fig. 6. Estimating the "true" parameters of the Vogelsberger system by comparing the differences in potential flattening on the left and the differences in the gradients of the potentials on the right. The comparisons are based on the distance interval from $0.5 \mathrm{up}$ to $2.0 \mathrm{kpc}$ (with steps of $0.05 \mathrm{kpc}$ ) from the centre of the galaxy. Left: mean absolute difference of the Vogelsberger potential flattening and the true potential flattening of the mock galaxy as a function of the flattening parameter $c / a$ of the Vogelsberger potential (black line). The grey horizontal line marks the positions where this difference has doubled with respect to the minimum 0.007 at $c / a \simeq 0.776$. Right: minimisation of the mean of the absolute differences in the gradients of the potential along the major and minor axis (compared to the mock galaxy) by varying the Vogelsberger model parameters $M_{1 \mathrm{kpc}}$ and $R_{\mathrm{s}}$. The figure is obtained after setting the flattening parameter to $c / a=0.776$. The colour bar is truncated at $5.0 \mathrm{~km} \mathrm{~s}^{-1}$. The green circle indicates the location at $\log _{10}\left(M_{1 \mathrm{kpc}}\left[M_{\odot}\right]\right) \simeq 7.69$ and $R_{\mathrm{s}}=4.9 \mathrm{kpc}$ where the differences are minimum $\left(\langle\Delta v\rangle_{\min }=0.31 \mathrm{~km} \mathrm{~s}^{-1}\right)$. Grey lines indicate the contours of constant mean absolute differences and are spaced by $1 \mathrm{~km} \mathrm{~s}^{-1}$. As a proxy for the error on the Vogelsberger parameters, a green contour is drawn where the differences are doubled with respect to the minimum difference.

On such an equipotential, it must hold that $\tilde{r}\left(R=0, z_{\Phi}\right)=$ $\tilde{r}\left(R_{\Phi}, z=0\right)$, and since $\tilde{r}$ only depends on $c / a$, then $q_{\mathrm{V}}(R, z)$ is independent of our mass parameter and scale radius ${ }^{6}$. We take values for $z_{\Phi}$ from 0.5 to $2.0 \mathrm{kpc}$ in steps of $0.05 \mathrm{kpc}$ along the minor axis and compute the corresponding $R_{\Phi}$ values (i.e. the radii where the equipotential contours that belong to $z_{\Phi}$ cross the major axis). For a given $c / a$, we then compute the mean of the absolute differences between the Evans mock galaxy potential flattening $(q=0.8)$ and the Vogelsberger potential flattening along the defined range for $z_{\Phi}$, that is, we compute: mean $\left(\left|q-q_{\mathrm{V}}\left(R=0, z_{\Phi}\right)\right|\right)$. We find that for $c / a \simeq 0.776$ this average difference is smallest (see left panel of Fig. 6). For our range of $z_{\Phi}$ and $c / a=0.776$, the Vogelsberger potential flattening increases almost linearly with $z_{\Phi}$, though the gradient is small $\left(0.018 \mathrm{kpc}^{-1}\right)$.

Given this value for the flattening parameter $c / a$, we proceed to obtain the corresponding values for the mass and scale radius of the mock galaxy, now described by the Vogelsberger profile. We do this by comparing $\left|R F_{R}\right| \equiv \sqrt{R\left|\frac{\partial}{\partial R} \Phi(R, z=0)\right|}$ along the major axis and $\left|z F_{z}\right| \equiv \sqrt{\left|z \frac{\partial}{\partial z} \Phi(R=0, z)\right|}$ along the minor axis with respect to their values for the mock galaxy. We investigate their trends for $R$ - and $z$-values identical to those used for $z_{\Phi}$ previously.

We vary the scale radius and the mass parameter $\log _{10}\left(M_{1 \mathrm{kpc}}\left[M_{\odot}\right]\right)$ and compute the mean of the absolute differences with respect to the mock galaxy obtained along the major and minor axis for $c / a=0.776$. We denote this by $\langle\Delta v\rangle \equiv \operatorname{mean}\left[0.5\left\{\operatorname{abs}\left(\Delta\left|R F_{R}\right|\right)+\operatorname{abs}\left(\Delta\left|z F_{z}\right|\right)\right\}\right]$. From the right panel of Fig. 6 we infer that $\langle\Delta v\rangle$ is minimum for mass param-

\footnotetext{
6 More precisely, $\tilde{r}$ depends on $r_{a}$ and $r_{E}(c / a)$, but we have chosen to fix the value of $r_{a}$ (and make it independent of $R_{\mathrm{s}}$ ).
}

eter $\log _{10}\left(M_{1 \mathrm{kpc}}\left[M_{\odot}\right]\right) \simeq 7.69$ and scale radius $R_{\mathrm{s}}=4.9 \mathrm{kpc}$ (green circle), although any value with $R_{\mathrm{s}} \geq 2 \mathrm{kpc}$ works well, as $\langle\Delta v\rangle$ does not vary strongly. To be able to compare these findings to the results from our Schwarzschild models (see Sect. 4.2.2), we estimate the error on these "true" parameters by considering those locations where $\langle\Delta v\rangle$ changes by a factor of two with respect to its minimum value (green contour). The mass parameter is then within the range [7.63, 7.73], the scale radius larger than $2.4 \mathrm{kpc}$. For the smaller scale radii $\left(R_{\mathrm{S}}<2 \mathrm{kpc}\right)$ slightly higher values for the characteristic mass parameter would be preferred, but $\langle\Delta v\rangle$ is also larger in such cases. We note that the NFW mass value that we estimated immediately above corresponds well with the mass enclosed within $1 \mathrm{kpc}$ of a spherical Evans model with $R_{\mathrm{c}}=1 \mathrm{kpc}$ and $v_{0}=20 \mathrm{~km} \mathrm{~s}^{-1}$ (as assumed in Sect. 2), since then $\log _{10}\left(M_{1 \mathrm{kpc} \text {,Evans }}\left[M_{\odot}\right]\right) \simeq 7.67$.

Although we do not constrain the flattening parameter, we can investigate how the parameters of our matched Vogelsberger model would change if different values for $c / a$ were taken. Setting $c / a=0.70$ results in $\langle\Delta v\rangle=0.32 \mathrm{~km} \mathrm{~s}^{-1}$ for its minimum at $\log _{10}\left(M_{1 \mathrm{kpc}}\left[M_{\odot}\right]\right) \simeq 7.69$ and $R_{\mathrm{s}}=4.4 \mathrm{kpc}$, and setting $c / a=$ 0.85 results in $\langle\Delta v\rangle=0.37 \mathrm{~km} \mathrm{~s}^{-1}$ for $\log _{10}\left(M_{1 \mathrm{kpc}}\left[M_{\odot}\right]\right) \simeq 7.69$ and $R_{\mathrm{S}}=5.1 \mathrm{kpc}$. Even for a spherical potential, i.e. $c / a=1.0$, we find the minimum $\langle\Delta v\rangle=0.62 \mathrm{~km} \mathrm{~s}^{-1}$ to be located at $\log _{10}\left(M_{1 \mathrm{kpc}}\left[M_{\odot}\right]\right) \simeq 7.68$ and $R_{\mathrm{s}}=6.1 \mathrm{kpc}$. The corresponding Vogelsberger mass parameter is thus not affected by the choice of $c / a$. The corresponding scale radius only increases slightly for larger values for the flattening parameter (i.e. rounder shapes), though the effect is rather small. In addition, the grey contours, which are drawn at fixed $\langle\Delta v\rangle$, span very similar regions for different values of $c / a$.

In Fig. 7 we compare the potential of the matched Vogelsberger model to the true Evans potential of our mock galaxy. In the left panel we show the gradients of the potentials along 

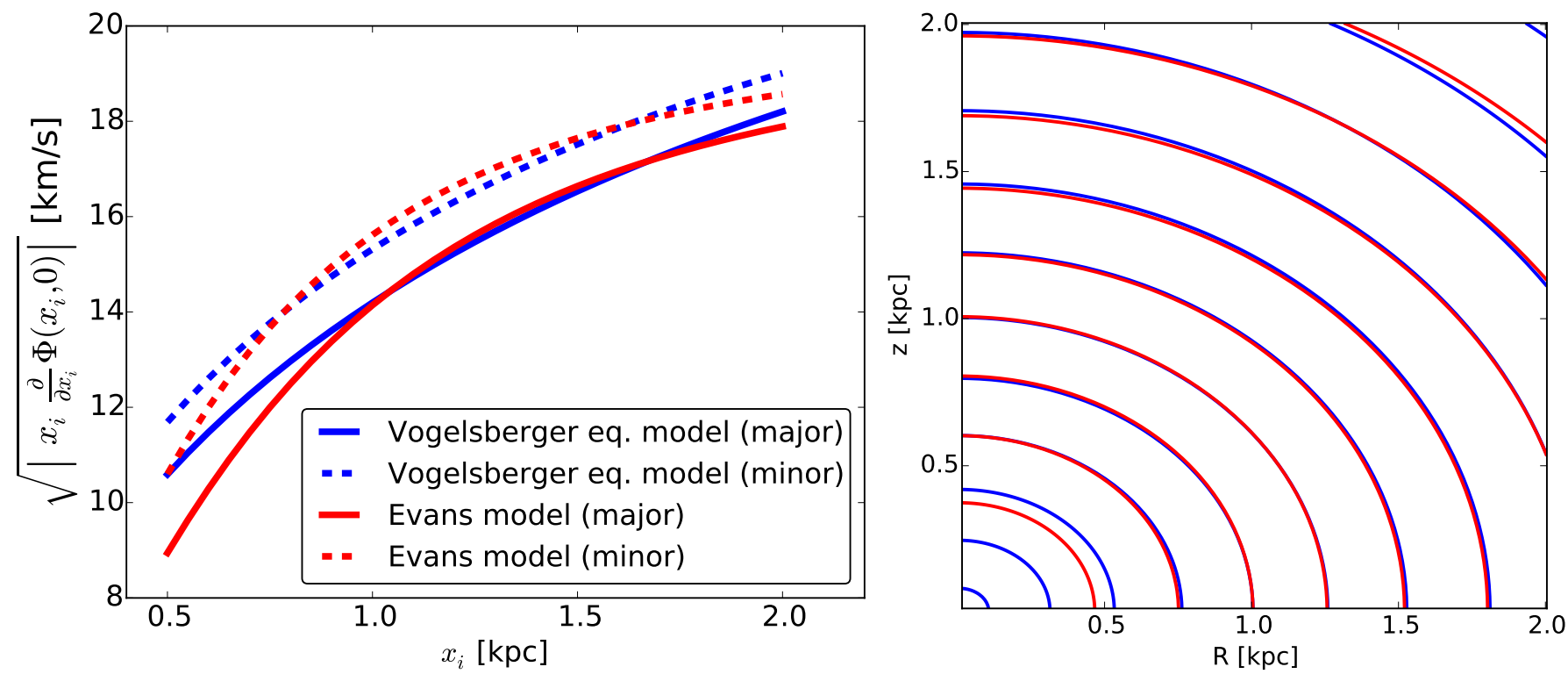

Fig. 7. Left: major (full lines) and minor (dashed lines) axis gradients of the potential as functions of $R$ and $z$, respectively, for the true Evans model (red) and for the matched Vogelsberger model with $c / a \simeq 0.776, R_{\mathrm{S}} \simeq 4.9 \mathrm{kpc}$ and $\log _{10}\left(M_{1 \mathrm{kpc}}\left[M_{\odot}\right]\right) \simeq 7.69$ (blue). Right: comparison of the isopotential contours for the true Evans model (red) and the matched Vogelsberger model (blue). For the purpose of this figure the zero-point of the potential is chosen here such that $\Phi_{\mathrm{E}}=\Phi_{\mathrm{V}}$ at $(R, z)=(1,0) \mathrm{kpc}$. For each potential, contours are drawn at the positions where $\Phi$ has changed in steps of $50 \mathrm{~km}^{2} \mathrm{~s}^{-2}$. In the region from $0.7 \mathrm{up}$ to $2 \mathrm{kpc}$ the matched Vogelsberger model follows well the true Evans potential. For more inner radii the (cusped) Vogelsberger models cannot reproduce the (less steep) cored behaviour of the Evans potential.

the major and minor axis. We note that the Evans model seems to have lower $\left|R F_{R}\right|$ and $\left|z F_{z}\right|$ for $R \lesssim 1 \mathrm{kpc}$ and $z \lesssim 0.75 \mathrm{kpc}$, respectively, than the matched NFW model. In the panel on the right we confirm that the potential flattening parameter is matched quite well by showing isopotential contours. Both panels reveal that only in the centre $(<0.7 \mathrm{kpc})$ and at distances larger than $3 \mathrm{kpc}$ do the gradients of both potentials start to deviate from each other.

In summary, the matched Vogelsberger system can be described by $\log _{10}\left(M_{1 \mathrm{kpc}}\left[M_{\odot}\right]\right) \simeq 7.69_{-0.06}^{+0.04}$ and by $R_{\mathrm{s}} \gtrsim 2.4 \mathrm{kpc}$ (with its most likely value at $R_{\mathrm{s}}=4.9 \mathrm{kpc}$ ) for $c / a=0.776$.

\subsubsection{Fitting Vogelsberger models with the Schwarzschild method: exploring different sample sizes}

Since we were not able to constrain the flattening parameter when the functional form of the potential of the system was known (see Sect. 4.1), we cannot expect to constrain the flattening parameter if we examine a different functional form for the model. We set $c / a=0.80$, which is equal to the observed axial ratio in the light, and subsequently find the inferences on the mass $\log _{10}\left(M_{1 \mathrm{kpc}}\left[M_{\odot}\right]\right)$ and scale radius $R_{\mathrm{s}}$. We initially make a grid in $\left(\log _{10}\left(M_{1 \mathrm{kpc}}\right), R_{\mathrm{S}}\right)$-space, where $R_{\mathrm{S}}$ ranges from 1 to $8 \mathrm{kpc}$ with steps of $\Delta R_{\mathrm{s}}=1 \mathrm{kpc}$, while for the characteristic mass we take steps of 0.05 for values from $\log _{10}\left(M_{1 \mathrm{kpc}}\left[M_{\odot}\right]\right)=7.55$ to $\log _{10}\left(M_{1 \mathrm{kpc}}\left[M_{\odot}\right]\right)=7.85$, that is, spanning a factor of only two in mass. Later, we also decided to sample $\log _{10}\left(M_{1 \mathrm{kpc}}\left[M_{\odot}\right]\right)=$ $[7.68,7.72]$ for $R_{\mathrm{S}} \in[1.5,7.5] \mathrm{kpc}$ with a similar $\Delta R_{\mathrm{S}}$ step.

To be more efficient we decrease the number of orbits compared to Sect. 4.1 and set $N_{\text {ener }}=24, N_{I_{2}}=24$, and $N_{I_{3}}=8$, such that a total of $24 \times 24 \times 8 \times 5^{3}=576000$ suborbits are integrated and $2 \times 24 \times 24 \times 8=9216$ orbital weights are determined. We find this gives good results in terms of recovery of the light profile and kinematics. In addition we also add regularisation terms to the fit in this more realistic experiment: by applying regularisation we set additional constraints such that the orbital weights are more smoothly distributed, that is, in a more physical way (as the weights relate to the distribution function, which itself is expected to be smooth). More details on the concept of regularisation and its effects can be found in Appendix C.

We present the results following the same structure of Sect. 4.1 and name the Vogelsberger models by MxxxRsyyy, where $\mathrm{xxx}=100 \log _{10}\left(M_{1 \mathrm{kpc}}\left[M_{\odot}\right]\right)$ and yyy $=100 R_{\mathrm{S}}($ in $\mathrm{kpc})$. We discuss how well we can recover the characteristic parameters of the Vogelsberger potential for mock datasets containing $10^{5}, 10^{4}$, and 2000 stars.

We start with the case of a kinematic dataset containing $10^{5}$ stars. We use $9 \times 9$ kinematic bins, but no folding. For this case, we find that model M772Rs250 provides the best fit $\left(\chi_{\text {tot }}^{2}=275.1\right)$. Figure 8 shows that this model accurately reproduces the mock velocity dispersions in all kinematic bins (since $\chi_{\text {kin }}^{2}=220.9$, which results in 0.68 per kinematic constraint). The fit is of comparable quality to the best-fit Evans model (for the same case) although the light is recovered slightly less well, which may be driven by the smaller number of orbits being used now, the choice of a different (and incorrect) potential form, or by adding regularisation in the fit.

Figure 9 shows the resulting $\Delta \chi^{2}$ distribution in $\left(\log _{10}\right.$ $\left.\left(M_{1 \mathrm{kpc}}\right), R_{\mathrm{s}}\right)$-parameter space. The scale radius of the Vogelsberger potential is constrained to $R_{\mathrm{S}}=2.5_{-0.1}^{+0.6} \mathrm{kpc}$ and the mass parameter to $\log _{10}\left(M_{1 \mathrm{kpc}}\left[M_{\odot}\right]\right)=7.72_{-0.01}^{+0.01}$. The Schwarzschild model thus prefers values towards the lower end for the scale radius and a mass parameter that agrees well with of our expectations. The panels on the right show the $\Delta \chi^{2}$-landscapes when only considering $\chi_{2 \mathrm{D} \text { light }}^{2}$ (top), $\chi_{\text {kin }}^{2}$ (top-middle), $\chi_{3 \mathrm{D} \text { light }}^{2}$ (bottom-middle), or $\chi_{\text {reg }}^{2}$ (bottom). The total $\Delta \chi^{2}$-landscape is dominated by the kinematics and 2D light.

As shown in Fig. 10, similar best-fit parameters are obtained for a smaller mock kinematic dataset containing $10^{4}$ stars and folding the data into $5 \times 5$ kinematic bins. The mass and scale parameters are constrained to $R_{\mathrm{s}}=3.0_{-0.4}^{+0.7} \mathrm{kpc}$ and 


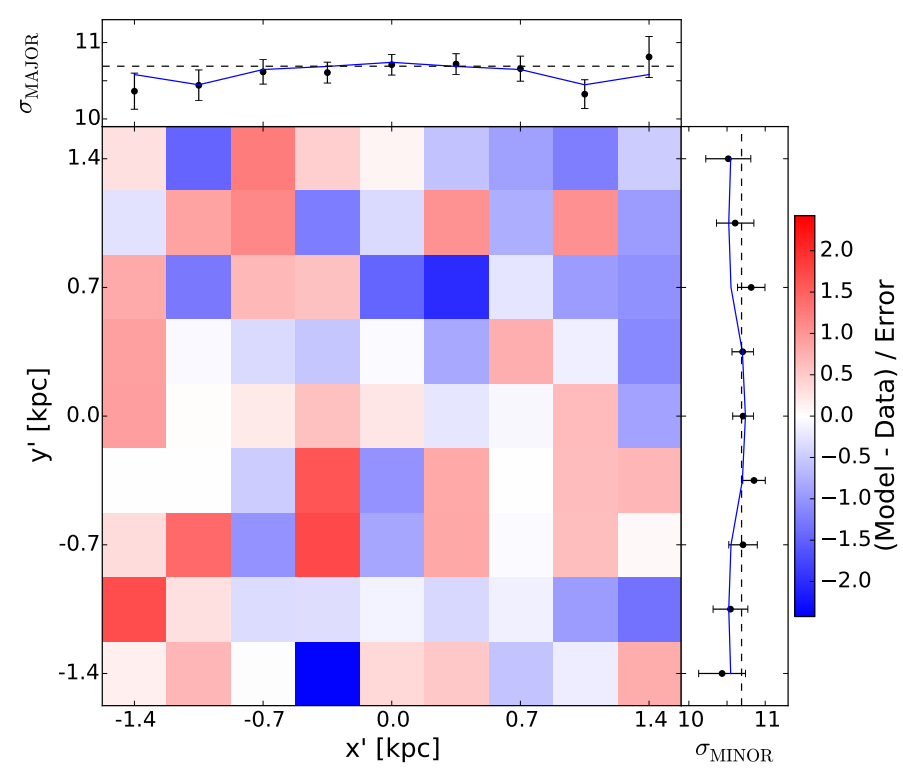

Fig. 8. Difference between the best-fit Vogelsberger model (M772Rs250, blue line in the subpanels) and the observed velocity dispersion when applying the Schwarzschild method in $9 \times 9$ kinematic bins to our mock dataset consisting of $10^{5}$ stars in the FOV (see Fig. 3 for a comparison).
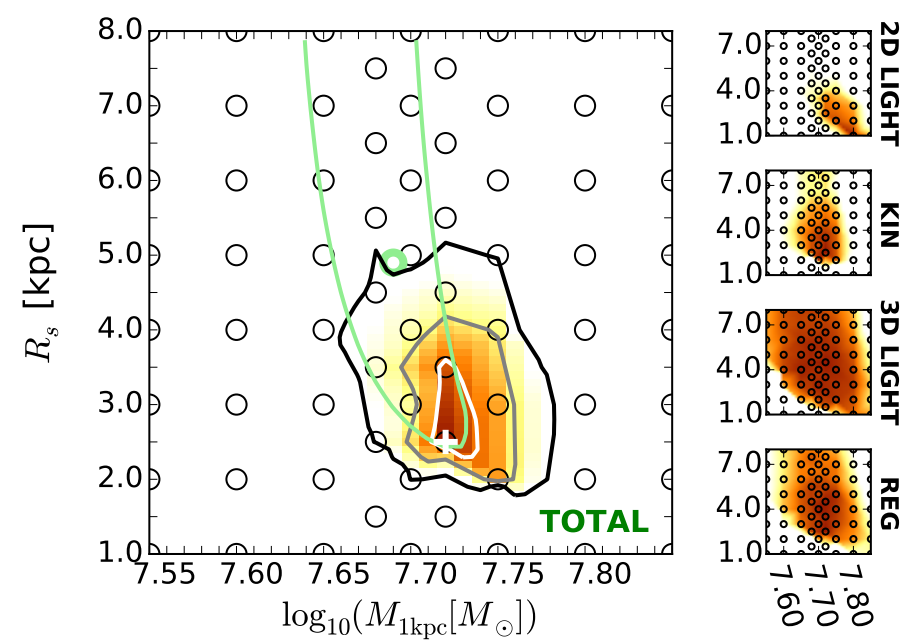

Fig. 9. Confidence intervals for the axisymmetric Vogelsberger model in $\left(\log _{10}\left(M_{1 \mathrm{kpc}}\right), R_{\mathrm{s}}\right.$ ) (after fixing $c / a=0.8$ ) for a kinematic dataset with $10^{5}$ stars and $9 \times 9$ kinematic bins. The $\Delta \chi^{2}=[2.3,6.18,11.8]-$ contours are in white, grey, and black, respectively. The best-fit model is indicated by the white cross, while the expectations are given by the green contour (identical to that shown in Fig. 6). The mass parameter is well constrained and models with $R_{\mathrm{s}} \leq 2.0 \mathrm{kpc}$ are strongly disfavoured, consistent with our expectations. The small panels on the right show the $\Delta \chi^{2}$ landscapes when only considering $\chi_{2 \mathrm{D} \text { light }}^{2}$ (top), $\chi_{\text {kin }}^{2}$ (top-middle), $\chi_{3 \mathrm{D} \text { light }}^{2}$ (bottom-middle), or $\chi_{\text {reg }}^{2}$ (bottom).

$\log _{10}\left(M_{1 \mathrm{kpc}}\left[M_{\odot}\right]\right)=7.75_{-0.03}^{+0.05}$. For the best-fit model M775Rs300, $\chi_{\text {tot }}^{2}=78.0$ and $\chi_{\text {kin }}^{2}=33.9$, or 0.339 per kinematic constraint on average. This $\chi_{\text {tot }}^{2}$ is lower than for the case of $10^{5}$ stars, likely because we folded the data. In comparison to the best-fit Evans model, the quality of the fit of the kinematics is slightly worse but is still very good.

When decreasing the sample size of the kinematics even further to 2000 stars, we find that models with low values for $R_{\mathrm{S}}$ and larger $\log _{10}\left(M_{1 \mathrm{kpc}}\left[M_{\odot}\right]\right)$ are now preferred, as shown
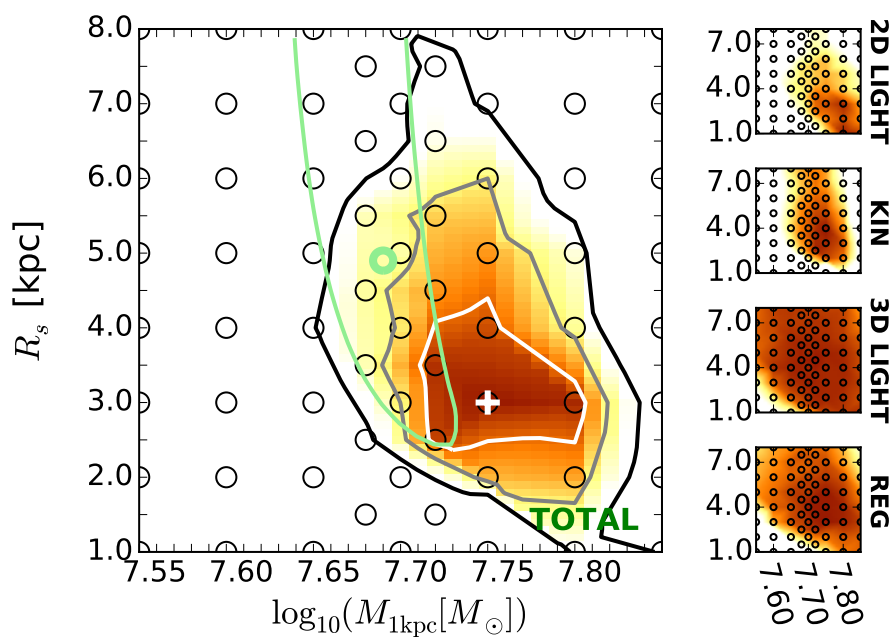

Fig. 10. Similar to Fig. 9, but now after fitting mock kinematic data consisting of $10^{4}$ stars and folding into $5 \times 5$ kinematic bins. The decrease in sample size (by a factor of ten) has led to a slight increase by the area spanned by the probability contours, although the inference on the mass parameter is still very good and only changed to slightly higher masses.
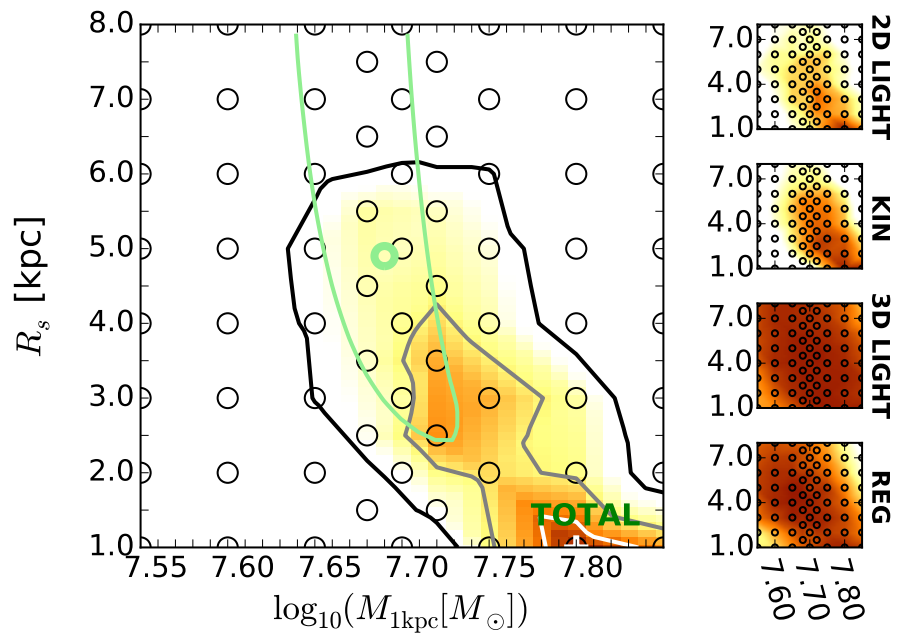

Fig. 11. As in Fig. 10, but now for a kinematic dataset with 2000 stars. We note how the confidence contours follow the shape of the green contour (derived in Fig. 6).

in Fig. 11, although the 95\%-confidence region still overlaps with the values for the parameters of the matched Vogelsberger model. For the best-fit model M780Rs100, we find $\chi_{\text {tot }}^{2}=101.9$ and $\chi_{\text {kin }}^{2}=67.2$, or 0.672 per kinematic constraint on average. We infer $R_{\mathrm{s}}=1.0_{-0.0}^{+0.2} \mathrm{kpc}$ and $\log _{10}\left(M_{1 \mathrm{kpc}}\left[M_{\odot}\right]\right)=7.80_{-0.01}^{+0.02}$ for the Vogelsberger parameters.

It is interesting to note that the shape of the confidence contours obtained from the Schwarzschild method for all sample sizes follows very closely the shape of the contours of $\langle\Delta v\rangle$ depicted in Fig. 6. We reiterate that the quantity $\langle\Delta v\rangle$ is a proxy for the difference in enclosed mass between the Evans and Vogelsberger models. This implies that the Schwarzschild method is actually very sensitive to enclosed mass, and is identifying the set of Vogelsberger models that best follow the true underlying mass distribution. Also interesting is that the trend favouring larger values of the mass parameter when decreasing sample size is present both for the Evans and the Vogelsberger models. 

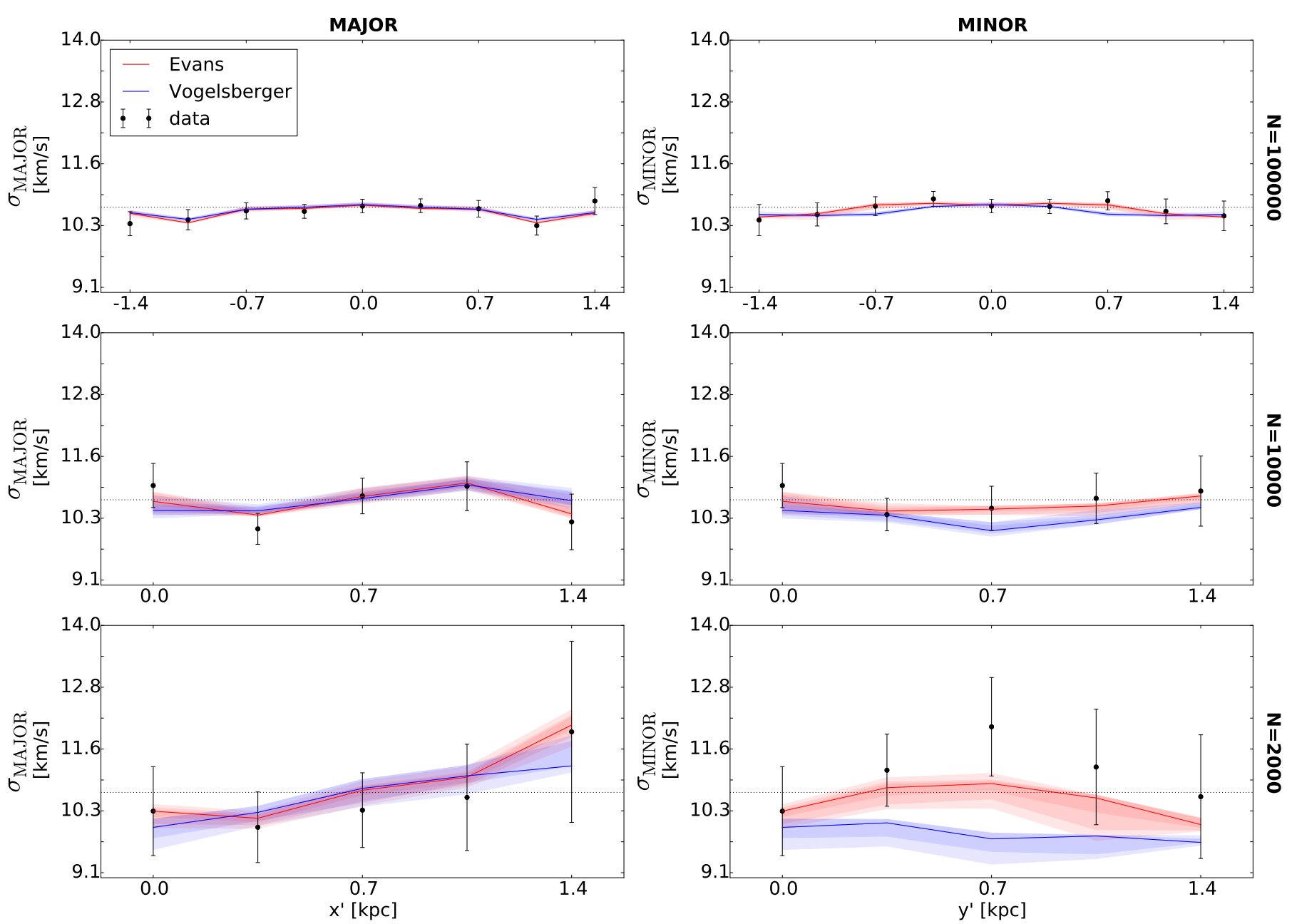

Fig. 12. Comparison of the results from the Schwarzschild modelling fits to the observed velocity dispersion along the major (left column) and minor (right column) axis. From top to bottom: best-fit Evans (red line) and Vogelsberger (blue line) models for kinematic datasets containing $N=10^{5}, N=10^{4}$, and 2000 stars, respectively. The shaded regions denote the error bands computed as described in the text. Black dotted lines indicate the input (theoretical, Eq. (4)) velocity dispersions.

We compare the Evans and Vogelsberger best-fit models to the observed velocity dispersions in Fig. 12. The left and right panels compare the behaviour on the major and minor axes, respectively, for different sample sizes for the kinematics: $10^{5}, 10^{4}$, and 2000 stars (in the top, middle, and bottom rows, respectively). The shaded areas enclose the minimum and maximum velocity dispersions for the evaluated models within the $\Delta \chi^{2}=[2.3,6.18,11.8]$-contours. These comparisons show that the Evans models fit the kinematics slightly better but that nearly equally good fits are provided by the Vogelsberger models (except in along the minor axis for the smallest dataset, bottom right panel).

From the analyses presented in this section we may thus conclude that the Schwarzschild modelling technique is sensitive to the mass enclosed and that it is successful in well constraining the mass parameter of the models, even if the functional form of the potential of the system is not known.

\section{Discussion and conclusions}

We explored the ability of Schwarzschild's orbit superposition method to characterise the intrinsic properties of an axisymmetric dSph galaxy, such as its mass, scale radius, and flattening. We did this by setting up an isothermal Sculptor-like mock galaxy that is flattened in both the luminous and dark components. We showed that Schwarzschild's method, applied to mock datasets with a realistic number of stars with measured radial velocities distributed following the luminosity profile of the system, is successful in recovering the characteristic mass parameter of the underlying (true) logarithmic potential, even if the potential flattening is not known. On the other hand, we find that we cannot put constraints on the flattening parameter.

Most likely, our inability to constrain the flattening is the consequence of our choice of the specific Evans model for our mock galaxy. In this model with a distribution function that is ergodic, the line-of-sight velocity profile is exactly the same everywhere and depends on the mass parameter only. This means that the kinematics are independent of the inclination and flattening, and the light alone does not contain enough information to constrain the flattening parameter.

One might also argue that it might not be optimal for a spectroscopic survey to sample stars according to the light profile of the system. In fact, slightly better results were obtained when the dataset with radial velocities provided an equal number of stars to each kinematic bin. In addition, just $\sim 30 \%$ of the light of the system is within our FOV for the specific Evans model used in this work. It might be possible that better results could be obtained with a more realistic and general distribution 
function (i.e. non-ergodic) applied to a galaxy for which the kinematic tracers cover the full system well and better sample the outskirts.

Since in reality the functional form of the potential of the system is not known, we also explored the case in which we assume an axisymmetric NFW model. We first determined the values of the parameters of the matched NFW model that mimics the mock galaxy best by comparing some basic properties (potential flattening and gradients in the potential). We found that even in this case, in which the orbits that form the building blocks of Schwarzschild's method are integrated in the incorrect potential, we can retrieve the correct characteristic mass and scale parameters.

We explored the dependencies of our results on the sizes of the data samples used, and find that a decrease in the number of stars with line-of-sight velocities only slightly affects the determination of the characteristic parameters of the model. For the smallest kinematic sample considered, with 2000 stars, the inference on the mass of the matched NFW model is somewhat poorer, but the true value differs by only $20 \%$ from the best-fit and also lies within the $95 \%$ confidence interval.

We checked that our results are not strongly dependent on the choices of the number of orbits in the orbit libraries, the number of kinematic or light bins, or the number of velocity bins, for example. Furthermore, we also briefly investigated the distribution functions for the the best-fit models, and found that, particularly when regularisation is included, they are quite similar to the distribution function of the mock $\mathrm{dSph}$ galaxy.

In conclusion, it is promising that the mass of our flattened system can be recovered to such a degree even if the flattening parameter is unknown. This is also aligned with the results of Kowalczyk et al. (2018), who applied their spherical Schwarzschild models on non-spherical objects. To some extent, this provides us with more confidence regarding previously reported estimates of the mass of dSph galaxies obtained assuming spherical symmetry.

Acknowledgements. We thank the anonymous referee whose comments helped to improve the manuscript. We also thank R. van den Bosch for supplying us the Schwarzschild code and L. Posti and P.T. de Zeeuw for many useful discussions regarding the project. A.H. acknowledges financial support from a VICI grant from the Netherlands Organisation for Scientific Research, NWO. For the analysis, the following software packages have been used: NumPy (Oliphant 2015), matplotlib (Hunter 2007), Jupyter Notebook (Kluyver et al. 2016).

\section{References}

Battaglia, G., Tolstoy, E., Helmi, A., et al. 2006, A\&A, 459, 423 Battaglia, G., Helmi, A., Tolstoy, E., et al. 2008a, ApJ, 681, L13
Battaglia, G., Irwin, M., Tolstoy, E., et al. 2008b, MNRAS, 383, 183 Battaglia, G., Tolstoy, E., Helmi, A., et al. 2011, MNRAS, 411, 1013 Battaglia, G., Helmi, A., \& Breddels, M. 2013, New A Rev., 57, 52 Binney, J., \& Tremaine, S. 2008, Galactic Dynamics, 2nd edn. (Princeton: Princeton University Press)

Boylan-Kolchin, M., Bullock, J. S., \& Kaplinghat, M. 2011, MNRAS, 415, L40 Breddels, M. A., \& Helmi, A. 2013, A\&A, 558, A35

Breddels, M. A., Helmi, A., van den Bosch, R. C. E., van de Ven, G., \& Battaglia, G. 2013, MNRAS, 433, 3173

Brooks, A. M., Kuhlen, M., Zolotov, A., \& Hooper, D. 2013, ApJ, 765, 22

Evans, N. W. 1993, MNRAS, 260, 191

Evans, N. W., An, J., \& Walker, M. G. 2009, MNRAS, 393, L50

Hayashi, K., \& Chiba, M. 2012, ApJ, 755, 145

Hayashi, K., \& Chiba, M. 2015, ApJ, 810, 22

Hayashi, K., \& Obata, I. 2019, MNRAS, in press, [arXiv:1902 .03054]

Hayashi, K., Ichikawa, K., Matsumoto, S., et al. 2016, MNRAS, 461, 2914

Hui, L. 2001, Phys. Rev. Lett., 86, 3467

Hunter, J. D. 2007, Comput. Sci. Eng., 9, 90

Irwin, M., \& Hatzidimitriou, D. 1995, MNRAS, 277, 1354

Jing, Y. P., \& Suto, Y. 2002, ApJ, 574, 538

Kim, S. Y., Peter, A. H. G., \& Hargis, J. R. 2018, Phys. Rev. Lett., 121, 211302

Kluyver, T., Ragan-Kelley, B., Pérez, F., et al. 2016, in Positioning and Power in Academic Publishing: Players, Agents and Agendas, eds. F. Loizides, \& B. Scmidt (IOS Press), 87

Klypin, A., Kravtsov, A. V., Valenzuela, O., \& Prada, F. 1999, ApJ, 522, 82

Kowalczyk, K., Łokas, E. L., \& Valluri, M. 2017, MNRAS, 470, 3959

Kowalczyk, K., Łokas, E. L., \& Valluri, M. 2018, MNRAS, 476, 2918

Magorrian, J. 2006, MNRAS, 373, 425

Massari, D., Breddels, M. A., Helmi, A., et al. 2018, Nat. Astron., 2, 156

Massari, D., Helmi, A., Mucciarelli, A., et al. 2019, A\&A, in press, https://doi.org/10.1051/0004-6361/201935613

Mateo, M. L. 1998, ARA\&A, 36, 435

McConnachie, A. W. 2012, AJ, 144, 4

Merrifield, M. R., \& Kent, S. M. 1990, AJ, 99, 1548

Moore, B., Ghigna, S., Governato, F., et al. 1999, ApJ, 524, L19

Navarro, J. F., Frenk, C. S., \& White, S. D. M. 1996, ApJ, 462, 563

Oliphant, T. E. 2015, Guide to NumPy, 2nd edn. (USA: CreateSpace Independent Publishing Platform)

Press, W. H., Teukolsky, S. A., Vetterling, W. T., \& Flannery, B. P. 1992, Numerical Recipes: The Art of Scientific Computing, 2nd edn. (New York, NY, USA: Cambridge University Press)

Richardson, T., \& Fairbairn, M. 2013, MNRAS, 432, 3361

Schwarzschild, M. 1979, ApJ, 232, 236

Schwarzschild, M. 1993, ApJ, 409, 563

Strigari, L. E., Koushiappas, S. M., Bullock, J. S., et al. 2008, ApJ, 678, 614

Strigari, L. E., Frenk, C. S., \& White, S. D. M. 2017, ApJ, 838, 123

van den Bosch, R. C. E., van de Ven, G., Verolme, E. K., Cappellari, M., \& de Zeeuw, P. T. 2008, MNRAS, 385, 647

Vera-Ciro, C. A., Sales, L. V., Helmi, A., \& Navarro, J. F. 2014, MNRAS, 439, 2863

Vogelsberger, M., White, S. D. M., Helmi, A., \& Springel, V. 2008, MNRAS, 385,236

Walker, M. G., Mateo, M., Olszewski, E. W., et al. 2007, ApJ, 667, L53

Walker, M. G., Mateo, M., \& Olszewski, E. W. 2009a, AJ, 137, 3100

Walker, M. G., Mateo, M., Olszewski, E. W., et al. 2009b, ApJ, 704, 1274

Walker, M. G., Olszewski, E. W., \& Mateo, M. 2015, MNRAS, 448, 2717

Wetzel, A. R., Hopkins, P. F., Kim, J.-H., et al. 2016, ApJ, 827, L23

Wolf, J., Martinez, G. D., Bullock, J. S., et al. 2010, MNRAS, 406, 1220

Zolotov, A., Brooks, A. M., Willman, B., et al. 2012, ApJ, 761, 71 


\section{Appendix A: Generating a mock kinematic dataset with realistic errors}

Like in Breddels et al. $(2013)^{7}$, we define $v_{i}$ as the true line-ofsight velocity of star $i$ and $\epsilon_{i}$ as the (true and unknown) measurement error on that star. Therefore, $v_{i}+\epsilon_{i}$ is the observed velocity of star $i$. We note that the expectation values for the moments of the measurement errors, which are drawn from a Gaussian distribution with $\sigma=2 \mathrm{~km} \mathrm{~s}^{-1}$, are given by: $E\left[\left\langle\epsilon_{i}^{n}\right\rangle\right]=E\left[\epsilon_{i}^{n}\right]=0$ for odd $n$ and $s_{n} \equiv E\left[\left\langle\epsilon_{i}^{n}\right\rangle\right]=E\left[\epsilon_{i}^{n}\right]=(n-1) ! ! \sigma^{n}$ for even $n$. In our terminology, $\hat{\mu}_{n}=E\left[\left\langle v_{i}^{n}\right\rangle\right]=E\left[v_{i}^{n}\right]$ denotes the true $n$th moment, $\mu_{n}$ is its estimator, and the observed $n$th moment is $m_{n}=\frac{1}{N} \sum_{i=1}^{N}\left(v_{i}+\epsilon_{i}\right)^{n}$ for a sample of $N$ stars in a given positional bin on the sky (i.e. kinematic bin).

Since we want to know the true value of the moments, that is, without measurement errors, we compute the estimators of the true moments. We also use raw moments (i.e. not taken about the mean velocities), and in what follows, we use the term "moments" to refer to "raw moments". Since we can only in practise compute the estimators of the true moments, we replaced $\hat{\mu}_{n}$ with $\mu_{n}$ in the right-hand side of the following equations. The first four moment estimators are then given by:

$\mu_{1}=\frac{1}{N} \sum_{i=1}^{N}\left(v_{i}+\epsilon_{i}\right)$

$\mu_{2}=\frac{1}{N} \sum_{i=1}^{N}\left(v_{i}+\epsilon_{i}\right)^{2}-s_{2}$,

$\mu_{3}=\frac{1}{N} \sum_{i=1}^{N}\left(v_{i}+\epsilon_{i}\right)^{3}-3 \mu_{1} s_{2}$,

and

$\mu_{4}=\frac{1}{N} \sum_{i=1}^{N}\left(v_{i}+\epsilon_{i}\right)^{4}-6 \mu_{2} s_{2}-3 s_{2}^{2}$.

To compute the error on these moments, we compute the square root of the variance of the moments;

$\operatorname{Var}\left(\hat{\mu}_{n}\right) \approx \operatorname{Var}\left(\mu_{n}\right) \approx \operatorname{Var}\left(m_{n}\right)=E\left[m_{n}^{2}\right]-\left(E\left[m_{n}\right]\right)^{2}:$

$$
\begin{aligned}
\operatorname{Var}\left(\mu_{1}\right) & =\frac{\mu_{2}+s_{2}-\mu_{1}^{2}}{N} \\
& =\frac{1}{N}\left\{\frac{1}{N} \sum_{i=1}^{N}\left(v_{i}+\epsilon_{i}\right)^{2}-\left[\frac{1}{N} \sum_{i=1}^{N}\left(v_{i}+\epsilon_{i}\right)\right]^{2}\right\}, \\
\operatorname{Var}\left(\mu_{2}\right) & =\frac{1}{N}\left[\mu_{4}-\mu_{2}^{2}+4 \mu_{2} s_{2}+2 s_{2}^{2}\right] \\
& =\frac{1}{N}\left\{\frac{1}{N} \sum_{i=1}^{N}\left(v_{i}+\epsilon_{i}\right)^{4}-\left[\frac{1}{N} \sum_{i=1}^{N}\left(v_{i}+\epsilon_{i}\right)^{2}\right]^{2}\right\},
\end{aligned}
$$

$$
\begin{aligned}
\operatorname{Var}\left(\mu_{3}\right)= & \frac{1}{N}\left[\mu_{6}+15 \mu_{4} s_{2}+45 \mu_{2} s_{2}^{2}+15 s_{2}^{3}-\mu_{3}^{2}\right. \\
& \left.-6 \mu_{3} \mu_{1} s_{2}-9 \mu_{1}^{2} s_{2}^{2}\right] \\
= & \frac{1}{N}\left\{\frac{1}{N} \sum_{i=1}^{N}\left(v_{i}+\epsilon_{i}\right)^{6}-\left[\frac{1}{N} \sum_{i=1}^{N}\left(v_{i}+\epsilon_{i}\right)^{3}\right]^{2}\right\},
\end{aligned}
$$

and

$$
\begin{aligned}
\operatorname{Var}\left(\mu_{4}\right)= & \frac{1}{N}\left[\mu_{8}+28 \mu_{6} s_{2}-\mu_{4}^{2}-12 \mu_{4} \mu_{2} s_{2}+204 \mu_{4} s_{2}^{2}\right. \\
& \left.-36 \mu_{2}^{2} s_{2}^{2}+384 \mu_{2} s_{2}^{3}+96 s_{2}^{4}\right] \\
= & \frac{1}{N}\left\{\frac{1}{N} \sum_{i=1}^{N}\left(v_{i}+\epsilon_{i}\right)^{8}-\left[\frac{1}{N} \sum_{i=1}^{N}\left(v_{i}+\epsilon_{i}\right)^{4}\right]^{2}\right\},
\end{aligned}
$$

where the errors on the third and fourth moment estimators also depend on:

$\mu_{6}=\frac{1}{N} \sum_{i=1}^{N}\left(v_{i}+\epsilon_{i}\right)^{6}-15 \mu_{4} s_{2}-45 \mu_{2} s_{2}^{2}-15 s_{2}^{3}$,

and

$\mu_{8}=\frac{1}{N} \sum_{i=1}^{N}\left(v_{i}+\epsilon_{i}\right)^{8}-28 \mu_{6} s_{2}-210 \mu_{4} s_{2}^{2}-420 \mu_{2} s_{2}^{3}-105 s_{2}^{4}$.

Clearly, the errors on the moments decrease when the number of stars in a kinematic bin increases.

\section{Appendix B: The effect of the sampling of line-of-sight velocities}

In the main text we drew samples of line-of-sight velocities that follow the light distribution of the mock galaxy. Here we show the results of applying the Schwarzschild modelling technique to a kinematic dataset consisting of $10^{5}$ stars, but this time distributed such that each kinematic bin has an equal number of stars, therefore effectively increasing the relative importance of the kinematic bins near the edges of our FOV with respect to the most central kinematic bins. This is because the errors on the moments decrease with increasing numbers of stars in a kinematic bin. In reality one might also consider probing larger radii.

Figure B.1 presents the inference on the mass and flattening parameter and should be compared to Figs. 2 and 3 . As can be observed, we have very similar inferences with the best-fit flattening parameter slightly moved into the direction of its input/true value. Nonetheless, this remains fairly unconstrained.

\footnotetext{
7 With respect to Breddels et al. (2013), small corrections to the equations are made. The updates concern Eqs. (A.4), (A.8), and (A.10).
} 

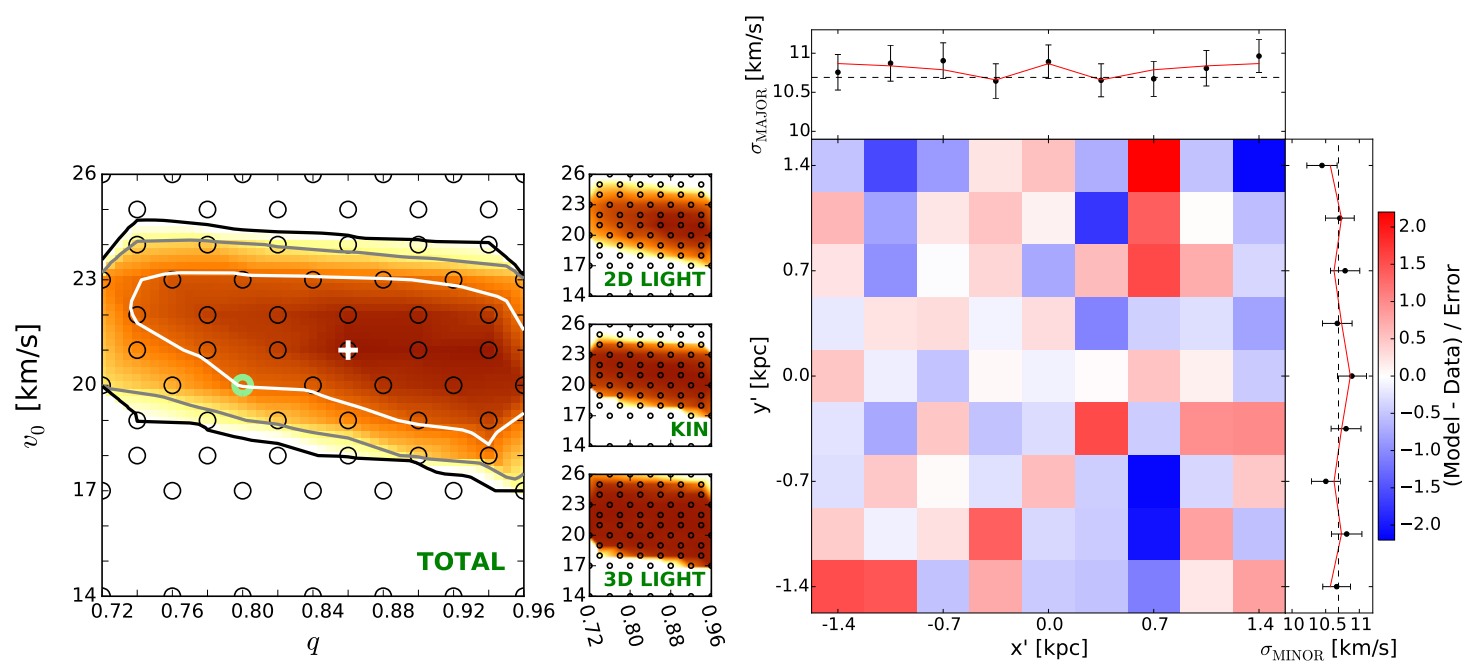

Fig. B.1. Left: confidence intervals after fitting the Evans models to a new realization of the dataset containing $10^{5}$ stars. This time we ensure an equal number of stars per kinematic bin. The inference on the mass parameter remains the same. The flattening parameter remains unconstrained but has slightly shifted into the direction of the correct flattening parameter. Right: velocity dispersion profile for the best-fit model q86v21.

\section{Appendix C: Regularisation}

The solution of our minimisation problem may result in a distribution of orbital weights that is rapidly varying or shows sharp discontinuities. Such a distribution would not be physical. Therefore we make the distribution of the orbital weights smoother by adding extra terms to the $\chi^{2}$-fitting algorithm such that a new quantity $\widetilde{\chi_{\text {tot }}^{2}}$ is minimised:

$\widetilde{\chi_{\text {tot }}^{2}}=\chi_{\text {tot }}^{2}+\chi_{\text {reg }}^{2}$.

This procedure is called regularisation. The regularisation strength is chosen such that the orbital weights are forced to change smoothly from one neighbouring orbit to the next, while finding similar values for the best-fit characteristic parameters. In addition, the confidence contours should not be significantly shaped by the $\chi_{\text {reg }}^{2}$-term. We refer the reader to van den Bosch et al. (2008) for more information about the exact implementation, in particular to Eqs. (28) and (29) of that paper. These equations require the $3 \mathrm{D}$ stellar density profile. For this work we assumed to know $\rho_{\text {lum }}$ (see Eq. (2)). In reality one needs the inclination angle to transform the observed surface brightness profile into the stellar density profile.

In the bottom panels of Fig. C.1, we show the effect of adding regularisation for the Evans q80v20 model (i.e. this is the true model) on the distribution of angular momentum around the symmetry axis $\left(L_{z}\right)$. The distributions can be compared to those of the q80v20 model without regularisation (top rows). Here we show the example with $10^{5}$ stars with line-of-sight velocities. The modelled distribution functions (blue) are generally smoother when regularisation is used. As a reference we also include the distributions for a realization of the mock galaxy (red). The model reproduces the mock distribution reasonably well, though some differences exist. The fact that only $\sim 30 \%$ of the total number of stars of the mock galaxy end up in our FOV might play a role here, in addition to the fact that we have discretized the data (by using kinematic bins, and by modelling only the first four velocity moments).
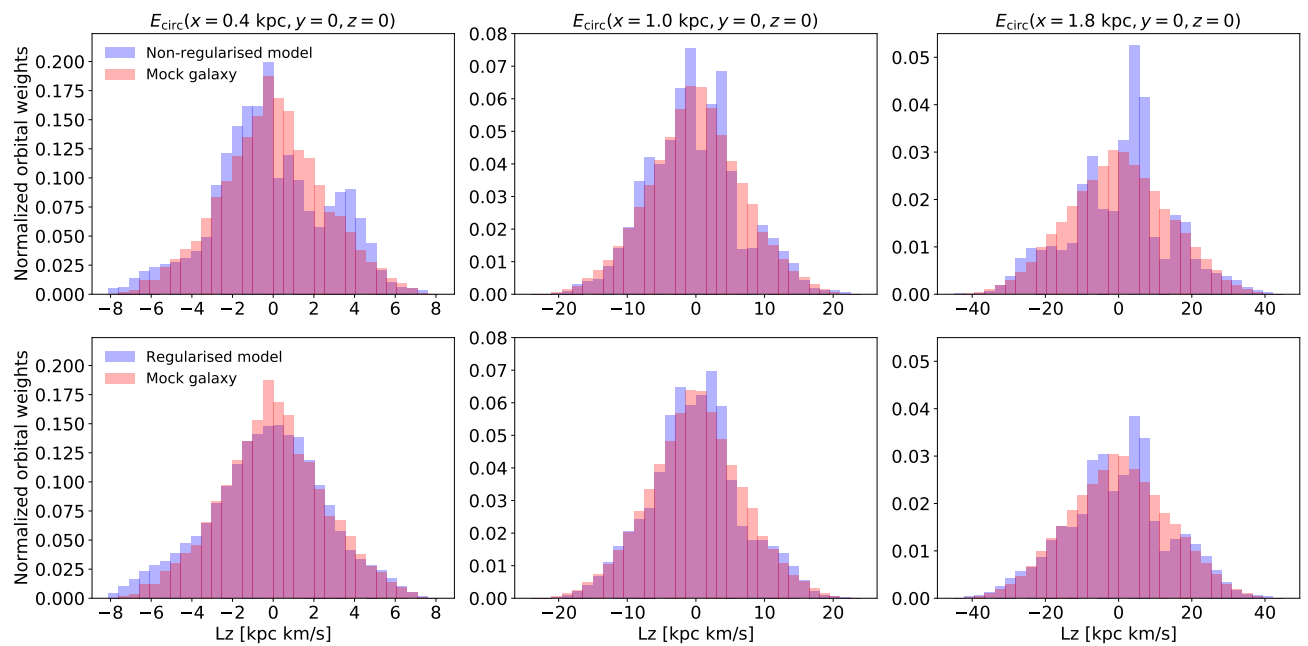

Fig. C.1. Orbital distributions of angular momentum around the symmetry axis, i.e. $L_{z}$, for fixed energy slices corresponding to circular orbits at $x=0.4$ (left), $x=1.0$ (middle), and $x=1.8 \mathrm{kpc}$ (right). We note the different axes ranges for the panels of each column. In the top row we show the distributions for the true Evans model q80v20 (blue) obtained after fitting a kinematic dataset of $10^{5}$ stars (see Sect. 4.1) and for a realization of the mock galaxy (red, here containing $4 \times 10^{5}$ stars in total). The effect of adding regularisation to the fit is shown in the bottom panels. Adding regularisation makes the recovered distribution smoother and more similar to the true distribution. 